\title{
INDEX PORTFOLIO AND WELFARE ANALYSIS UNDER HETEROGENEOUS BELIEFS
}

\author{
XUE-ZHONG HE AND LEI SHI \\ University of Technology Sydney \\ UTS Business School, Finance Discipline Group \\ PO Box 123 Broadway \\ NSW 2007, Australia
}

\begin{abstract}
With a growing popularity of index funds, we adopt a differences-in-opinion, general equilibrium framework to examine theoretically whether investors are better off with an index portfolio than active investing. In contrary to the conventional view, we find that, even for an active investor with the most accurate belief, switching to an index portfolio can significantly improve his expected ex-post welfare when the active investors have incorrect beliefs or face incomplete information. Moreover, the welfare improvement becomes more substantial when the active investors are more risk averse.
\end{abstract}

JEL Classification: G11, G12, D84.

Keywords: Index funds; Active investing; Welfare analysis; Differences in opinion; General equilibrium model.

Current version: August 24, 2016.

We would like to thank Doug Foster and Qiaoqiao Zhu for their comments and participants of the 2012 Asian FA conference in Taipei, in particular Qunzi Zhang (discussant). An earlier version of this work has been circulated under the title "Heterogeneous Beliefs and the Performances of Optimal Portfolios". The financial support from the Australian Research Council (ARC) under the Discovery Grant (DP130103210), the National Natural Science Foundation of China Grant (NSFC: 71320107003), and the UTS Business School research grant is gratefully acknowledged. The usual caveats apply.

Corresponding author: Lei Shi, Finance Discipline Group, UTS Business School, University of Technology Sydney, PO Box 123 Broadway, NSW 2007, Australia. Email: Lei.Shi@uts.edu.au. Ph: (61 2) 9514 1972. Fax: (61 2) 95147711 . 


\section{INTRODUCTION}

Since Fargo Bank launched the first index fund in the 1970s (Sharpe 2007), we have seen a growing popularity of index funds. According to Cremers, Ferreira, Matos and Starks (2011), "Nearly 28,000 equity funds and \$10.5 trillion in assets under management were recorded as of December 2010 (Investment Company Institute (2011)). The percentage invested in explicitly indexed funds has grown rapidly over the last decade from about $14 \%$ of assets under management in 2002 to about $22 \%$ in 2010." Moreover, apart from the explicitly indexed funds, there are also closet index funds, which claim to practice active management, but stay close to the benchmark index. Petajisto (2013) shows that out of 1,124 mutual funds between 1990-2009, 180 funds are categorized as closet indexers. Index funds are generally perceived as a low-cost alternative to active management, Dyck, Lins and Pomorski (2013) report a difference in cost of $0.35 \%$ per year between active and passive management. Therefore, before cost, active management on average performs approximately the same as passive management. This observation is consistent with a modified version of the Index Fund Premise in Sharpe (2007), originally used by Fargo Bank, saying that, "Few of us are as smart as all of us, it is hard to identify them in advance, and they may charge more than they are worth."

Empirical evidence suggests that investors spent a significant amount on the cost of active investing. According to French (2008), averaging over 1980-2006, investors in the U.S "spend $0.67 \%$ of the value of all NYSE, Amex, and NASDAQ stocks each year trying to beat the market". Although, on average, mutual funds in the U.S. tend to underperform the S\&P500르, there is evidence that mutual funds are able to outperform their benchmarks when they are highly active $^{2}$ and when financial markets are less efficient ${ }^{3}$. More importantly, there are evidences that sophisticated investors earn positive returns on new cashflows both into and out of the funds

\footnotetext{
${ }^{1}$ The early work by Jensen (1968) find that in the 1945-1964 period, mutual funds were on average not able to outperform the market, even when fund returns are measured before management expenses. Moreover, looking at unadjusted returns, Gruber (1996) reports, in the 1985-1994 period, that mutual funds underperformed the market by $1.94 \%$ per year. More recently, Busse, Goyal and Wahal (2010) examine the performance of 4,617 active domestic equity institutional products between 1991 and 2008. After controlling for the Fama and French (1993) factors plus momentum, they find that the four-factor alpha is statistically indistinguishable from zero. Furthermore, Dyck et al. (2013) also report an underperformance of $0.28 \%$ per year by active management after cost based on the U.S. and Canadian defined benefit pension plans equity holdings over the 1993-2008 period. ${ }^{2}$ Cremers and Petajisto (2009) find that funds with the highest Active Share, which measures the share of portfolio holdings that differ from the benchmark, outperform their benchmark by $1.51-2.40 \%$ per year. Cremers et al. (2011) also find that Active Share predicts funds' future performance when examining the equity mutual funds world wide.

${ }^{3}$ Dyck et al. (2013) document that in the EAFE equity markets, net-of-cost active outperformance is 49 bps per year, while in the less efficient emerging markets the active outperformance is a substantial 246 bps per year.
} 
(Gruber 1996) and they tend to increase allocations to active management in the less efficient markets, where active strategies work better (Dyck et al. 2013). Therefore, the general perception is that, although active investing is a negative sum game (French 2008), the group of sophisticated investors can benefit from it at the expense of unsophisticated investors or clienteles. Hence, the sophisticated investors should keep pursuing active management in trying to beat the market and the unsophisticated investors should switch to indexing.

This paper considers active investors who are subjective utility maximizers and an index investor who buys and holds the market portfolio of all risky stocks. In contrary to the conventional view, we show that, when active investors do not know the true probabilities either due to biases or incomplete information, even for the investor with the most accurate belief, active investing can be harmful with large welfare costs. Therefore the potential welfare improvement by switching to an index portfolio can be quite substantial.

In this paper, we adopt the differences-of-opinion (DO) general equilibrium framework that has been widely used in the literaturet. In DO models, agents have symmetric information and agree to disagree about the underlying model of economic fundamentals. Each agent believes in his interpretation of the observed information and ignores the interpretations of others. Essentially, the DO models "highlight aspects of the world that investors still disagree about after they have learned all they can from each other" (Banerjee and Kremer 2010) and therefore should be distinguished from rational expectation (RE) models of asymmetric informations. Banerjee (2011) develops an empirical method to distinguish between the RE and DO models.

More specifically, we build a general equilibrium model of a financial market in continuoustime with active investors who disagree about the drift terms of the dividend processes, either due to biases or incomplete information. The disagreement motivates the investors to engage in speculation with each other. The outcome of their speculation depends on whose belief is more accurate, i.e. closer to the truth. The investors maximize their life-time utility of the consumption under their subjective probability measures. This means that the expected

\footnotetext{
${ }^{4}$ See, for example, Detemple and Murthy (1994), Zapatero (1998), Basak (2000, 2005), Anderson, Ghysels and Juergens (2005), Buraschi and Jiltsov (2006), Jouini and Napp (2006, 2007, 2011), Li (2007), David (2008), Berraday (2009), Dumas, Kurshev and Uppal (2009), Xiong and Yan (2010), Yan (2010), Cvitanić, Jouini., Malamud. and Napp (2012), Fedyk, Heyerdahl-Larsen and Walden (2013), Bhamra and Uppal (2014), Hansen (2015) and Atmaz and Basak (2015).

${ }^{5}$ In this class of models, uninformed agents try to extract information from currently observable price; see, for example, Grossman and Stiglitz (1980), Admati (1985), Wang (1993), Watanabe (2008) and Biais, Bossaerts and Spatt (2010).
} 
utilities of the investors generally do not coincide with the expected utilities under the objective probability measure. Following Fedyk et al. (2013), we use the objective expected utilities as a measure for the expected ex-post welfare.

Apart from the active investors, there is also an index investor who holds the market portfolio and consumes a fraction of the aggregate dividend. The DO models typically do not consider index investors for two reasons. Firstly, index investing is in general not an optimal strategy for investors under their subjective beliefs. Secondly, when index investors consume a constant fraction of the aggregate dividend, they do not affect the equilibrium prices. One exception is Chabakauri and Rytchkov (2014) who constrain one group of agents to invest only in an index and a risk-free asset. When agents have a homogeneous belief, they show that the indexing actually leads to a welfare reduction instead of improvement. Moreover, the welfare reduction of the index investing is minimal in Chabakauri and Rytchkov (2014), "in our calibration of the model the investor would not give up more than $0.15 \%$ of his wealth for the possibility to trade all assets individually. Thus, indexing does not make investors notably worse off and in the absence of other market frictions it can be a viable trading strategy." In contrast, we show that when the active investing can have large welfare costs to investors, an indexing portfolio can significantly improve the expected ex-post welfare.

Following Fedyk et al. (2013), we consider two active investors, $A$ and $B, A$ has more accurate belief than $B$, in addition to an index investor $I$. The expected ex-post welfare achieved by the active and index investors is denoted by $\mathcal{U}_{i}$ for $i \in\{A, B, I\}$. From the extensive literature on the market selection hypothesis, eventually investor $A$ with a more accurate belief will drive investor $B$ to extinction and thus will have a higher consumption growth than the index portfolio in the long run 6 . Therefore, conventional wisdom would suggest that the expected ex-post welfare should be ranked as $\mathcal{U}_{B}<\mathcal{U}_{I}<\mathcal{U}_{A}$, which means investor $A$ is better off than the index investor who is better off than investor $B$. This is obviously true when $A$ 's belief coincides with the truth. However, when the objective and subjective beliefs differ, we show under certain conditions, quite remarkably, that the welfare ranking becomes $\mathcal{U}_{\mathcal{B}}<\mathcal{U}_{A}<\mathcal{U}_{I}$, indicating that the index portfolio dominates the active investing.

\footnotetext{
${ }^{6}$ See, for example, Sandroni (2000), Blume and Easley (2006, 2009a, 2009b), Kogan, Ross, Wang, Westerfield (2006, 2012), Yan (2008), Muraviev (2013), Cvitanić and Malamud (2011), Branger, Schlag and Wu (2015).
} 
In Section 2, we present our baseline model with logarithmic utility and constant heterogeneous beliefs. Here, both active investors have incorrect beliefs. In this case, the welfare ranking depends on two quantities: the absolute bias ratio $0<\vartheta \leq 1$ and the bias correlation $-1 \leq \rho \leq 1$. The former measures the relative accuracy of investors' beliefs and the latter measures whether investors make similar or opposite mistakes about the expected dividend returns. We find that a larger $\vartheta$ close to 1 and a more negative $\rho$ close to -1 favor the index investor and the welfare ranking $\mathcal{U}_{B}<\mathcal{U}_{A}<\mathcal{U}_{I}$ is more likely to prevail. This implies that, although the active investor with a more accurate belief almost surely achieve a higher consumption growth in the long run, he may be worse off than the index investor in terms of the objective expected utilities. This happens when the belief of investor $A$ is not much more accurate and opposite to the investor with a less accurate belief. Intuitively, the active investors trade for both speculative and risk-sharing purposes, the former is caused by disagreement and the latter is for consumption-smoothing. However, their erroneous beliefs distort the optimal balance between speculation and risk-sharing, which hurts their welfare. Moreover, the opposite beliefs lead to larger disagreement, which makes their consumption paths more volatile and amplifies the detrimental effect of the erroneous beliefs on the welfare. Hence, in this case, even the investor with a more accurate belief achieves a lower welfare than the index investor. In a simple calibration with plausible parameter values, even when the absolute belief biases are relatively small, we find that the index portfolio can improve the welfare by $20 \%$ for $\vartheta=1$, that is, when the active investors are equally biased.

In Section 3, we extend our analysis to CRRA utility with relative risk aversion $\gamma>0$. Apart from obtaining the same results and intuition to the baseline model, we find that an increase in the risk aversion has two offsetting effects on investors' welfare. On the one hand, it increases the concavity of the utility function, which magnifies the negative impact caused by incorrect beliefs. On the other hand, it reduces speculation and makes investors' consumption shares less volatile, which mitigates the aforementioned negative impact. We find that the first effect tends to dominate the second effect when the risk aversion is below a threshold, above which the two effects offset each other. Therefore, in general a higher risk aversion works in the favor of the index investor. With the same parameter values as in the baseline case, we show that, when $\gamma=2$, the index portfolio can improve the welfare by more than $50 \%$ compared to the active investing, which is more than double the amount in the logarithmic case with $\gamma=1$. 
We consider the impact of learning in Section 4. Due to the loss of tractability, we use Monte Carlo simulations to evaluate the objective expected utilities of the active and index investors. Within this setting, even a perfectly rational investor's belief can deviate from the truth due to incomplete information. We find that in the case of constant drifts, the welfare ranking depends critically on the prior variance of the investor who learns about the drifts. We find that a larger prior variance tends to favor the index investor in terms of the welfare. In a complex learning environment where the drift rates follow mean-reverting processes and the investors receive a public signal but disagree about its informativeness, the index portfolio dominates the active investing when the active investors have the opposite interpretation of the signal (i.e. one interprets the signal as good news and other interprets it as bad news). In Section 5, we consider a more realistic scenario where the consumption growth of the index portfolio is more volatile than the aggregate consumption growth. We conclude the paper in Section 6 and Appendix A contains all the proofs.

\section{The Model And Welfare Analysis}

Following Jouini and Napp (2007), Yan (2008), Dumas et al. (2009), and Fedyk et al. (2013), we consider a dynamic equilibrium model with two active investors who trade speculatively with each other due to heterogeneity in beliefs, and an index investor who holds the market portfolio of risky securities and consumes a constant fraction of the aggregate dividend]. Under the assumptions of logarithmic utility and constant beliefs 8 , this section provides sufficient conditions under which the index investor achieves a better welfare under the objective probability measure than the active investors.

2.1. Information Structure and the Securities Market. Consider a continuous-time pureexchange economy with a single consumption good. The uncertainty is represented by a filtered probability space $(\Omega, \mathcal{F},\{\mathcal{F}(t)\}, \mathcal{P})$ on which a $K$-dimensional Wiener process $\mathbf{Z}(t)=$

\footnotetext{
${ }^{7}$ In Section 5, we consider the case in which the index portfolio differs from the market portfolio, and it has a more volatile consumption growth than that of aggregate endowment.

${ }^{8}$ In the baseline model, for simplicity we assume beliefs do not update over time. The cost of relaxing this assumption is the loss of tractability. In Section 4, we use Monte Carlo simulations to examine the impact of learning on welfare.
} 
$\left(Z_{1}(t), \cdots, Z_{K}(t)\right)^{\top}$ is defined. Let $\{\mathcal{F}(t)\}$ be the augmented filtration generated by $\mathbf{Z}(t)$. For simplicity, the Wiener processes are assumed to be uncorrelated?

There are $K$ risky assets (stocks) and one risk-free bond. The price of the risk-free bond $B(t)$ follows

$$
d B(t)=r(t) B(t) d t, \quad B(0)=1
$$

where $r(t)$ is the risk-free interest rate. The dividend $D_{k}(t)$ of stock $k$ follows

$$
d D_{k}(t)=D_{k}(t)\left[\mu_{k} d t+\sigma_{k} d Z_{k}(t)\right], \quad k=1,2, \cdots, K
$$

with constant $\mu_{k}$ and $\sigma_{k}$. Let $S_{k}(t)$ be the ex-dividend price of stock $k$. Denote the instantaneous return of stock $k$ as $d R_{k}(t) \equiv \frac{d S_{k}(t)+D_{k}(t) d t}{S_{k}(t)}$. The vector of instantaneous stock returns is given by

$$
d \mathbf{R}(t) \equiv\left(d R_{1}(t), \cdots, d R_{K}(t)\right)=\boldsymbol{\mu}_{S}(t) d t+\boldsymbol{\sigma}_{S}(t) d \mathbf{Z}(t)
$$

where $\boldsymbol{\mu}_{S}(t)$ is the vector of expected returns under $\mathcal{P}$ and $\mathbf{V}_{S}(t) \equiv \boldsymbol{\sigma}_{S}(t) \boldsymbol{\sigma}_{S}(t)^{\top}$ is the covariance matrix at time $t$. By assuming a complete market, there exists a unique state price density $\xi(t)$ with initial value $\xi(0)=1$ and

$$
d \xi(t)=-\xi(t)\left[r(t) d t+\boldsymbol{\kappa}(t)^{\top} d \mathbf{Z}(t)\right]
$$

where $\boldsymbol{\kappa}(t)=\boldsymbol{\sigma}(t)^{-1}\left(\boldsymbol{\mu}_{S}(t)-r(t) \mathbf{1}\right)$ denotes the vector of the market prices of risk. We assume that the riskless bond $B$ is in zero net supply and each risky asset $S_{k}$ has $N_{k}$ shares available. Therefore, the aggregate dividend or endowment process is given by

$$
D(t)=\sum_{k=1}^{K} N_{k} D_{k}(t) .
$$

2.2. Investors. There are two active investors $A$ and $B$ who are subjective utility maximizers with probability measures $\mathcal{P}_{A}$ and $\mathcal{P}_{B}$, respectively 10 . Investors $A$ and $B$ have constant beliefs about the mean dividend growth rates, denoted by $\mu_{k}^{i}$ for $i \in\{A, B\}$ and $k=1, \cdots, K$. From

\footnotetext{
${ }^{9}$ As in Yan (2008) and Fedyk et al. (2013), we can consider correlated Wiener processes with a constant correlation matrix; however this does not affect our results.

${ }^{10}$ When investors have homogeneous preference and heterogeneous beliefs, following the market selection literature (Yan (2008) and Fedyk et al. (2013)), only the investor with the most accurate belief can survive in the long-run. Therefore, to conduct a welfare analysis, we consider a parsimonious model of two investors with homogeneous preference and compare their relative performance to an index portfolio.
} 
investor $i$ 's point of view, the dividend processes evolve according to

$$
d D_{k}(t)=D_{k}(t)\left[\mu_{k}^{i} d t+\sigma_{k} d Z_{k}^{i}(t)\right]
$$

where

$$
d Z_{k}^{i}(t)=d Z_{k}(t)-\theta_{k}^{i} d t \text { with } \theta_{k}^{i} \equiv \frac{\mu_{k}^{i}-\mu_{k}}{\sigma_{k}} .
$$

Therefore, both the active investors have incorrect beliefs. Parameter $\theta_{k}^{i}$ measures investor $i$ 's optimism/pessimism about stock $k$ 's dividend growth, which is persistent as they do not update their beliefs over time 11 . Therefore, to be consistent, under investor $i$ 's belief, the stock returns evolve according to

$$
d \mathbf{R}(t)=\boldsymbol{\mu}_{S}^{i}(t) d t+\boldsymbol{\sigma}_{S}(t) d \mathbf{Z}_{i}(t)
$$

where $\boldsymbol{\mu}_{S}^{i}(t)=\boldsymbol{\mu}_{S}(t)+\boldsymbol{\sigma}_{S}(t) \boldsymbol{\theta}_{i}$ is the vector of expected returns perceived by investor $i$. By Girsanov's theorem, the subjective probability belief $\mathcal{P}_{i}$ can be characterized by

$$
M_{i}(t) \equiv \frac{d \mathcal{P}_{i}}{d \mathcal{P}}=\exp \left\{-\frac{1}{2} \boldsymbol{\theta}_{i}^{\top} \boldsymbol{\theta}_{i} t+\boldsymbol{\theta}_{i}^{\top} \mathbf{Z}(t)\right\}, \quad i \in\{A, B\}
$$

where $\boldsymbol{\theta}_{i}=\left(\theta_{1}^{i}, \theta_{2}^{i}, \cdots, \theta_{K}^{i}\right)^{\top}$. The length of the vector $\boldsymbol{\theta}_{i}$,

$$
\left\|\boldsymbol{\theta}_{i}\right\| \equiv \sqrt{\boldsymbol{\theta}_{i}^{\top} \boldsymbol{\theta}_{i}}, \quad i \in\{A, B\}
$$

measures investor $i$ 's absolute belief bias, which is the total distance between $\mathcal{P}_{i}$ and $\mathcal{P}$.

There are two important quantities for deriving our results under the constant beliefs. One is the absolute bias ratio between the investors and the other is their bias correlation. We define these two quantities in the following.

Assumption 2.1. Investors $A$ and $B$ have different subjective beliefs, i.e., $\boldsymbol{\theta}_{A} \neq \boldsymbol{\theta}_{B}$, and the absolute belief biases are strictly positive and satisfy $\vartheta \leq 1$, where

$$
\vartheta \equiv \frac{\left\|\boldsymbol{\theta}_{A}\right\|}{\left\|\boldsymbol{\theta}_{B}\right\|}
$$

is the absolute bias ratio between investors $A$ and $B$.

\footnotetext{
${ }^{11}$ We examine the impact of learning in Section 4.

${ }^{12}$ Note that the absolute belief bias defined here is equivalent to the survival index in Yan (2008) and the transfer index in Fedyk et al. (2013) when investors have homogeneous preference.
} 
Under assumption 2.1, both the investors have biased beliefs and investor $A$ is either equally biased as or less biased than $B$. Next, we define the bias correlation between the investors as follows.

Definition 2.2. The bias correlation between investors $A$ and $B$ is defined by

$$
\rho \equiv \frac{\boldsymbol{\theta}_{A}^{\top} \boldsymbol{\theta}_{B}}{\left\|\boldsymbol{\theta}_{A}\right\|\left\|\boldsymbol{\theta}_{B}\right\|}, \quad \text { where }-1 \leq \rho \leq 1 .
$$

According to Definition 2.2, the bias correlation measures the linear dependence between the beliefs of the investors. When $\rho=1$, investors $A$ and $B$ either both underestimate or overestimate the drifts of the dividend processes, i.e. $\theta_{k}^{A} \theta_{k}^{B}>0$, for $k=1, \cdots, K$. In contrast, $\rho=-1$ indicates that $\theta_{k}^{A} \theta_{k}^{B}<0$ for all stocks. In this case, one of the investors underestimates while the other overestimates the drifts. Moreover, $\rho=0$ implies that investors' belief biases are linearly independent. Furthermore, the bias correlation can be expressed as $\rho=\cos (\varpi)$, where $\varpi$ is the angle between vectors $\boldsymbol{\theta}_{A}$ and $\boldsymbol{\theta}_{B}$. For example, $\varpi=0, \frac{\pi}{2}, \pi$ correspond to $\rho=1,0,-1$ respectively 13 .

In our baseline model, we assume logarithmic utility. An extension to CRRA utility is given in Section 3. The objective of the investors is to maximize

$$
\mathbb{E}_{0}^{i}\left[\int_{0}^{\infty} e^{-\beta t} \ln \left(c_{i}(t)\right) d t\right]=\mathbb{E}_{0}\left[\int_{0}^{\infty} e^{-\beta t} M_{i}(t) \ln \left(c_{i}(t)\right) d t\right], \quad i \in\{A, B\},
$$

subject to the dynamic budget constraint

$$
d W_{i}(t)=\left(W_{i}(t)\left(r(t)+\left(\boldsymbol{\pi}_{i}(t)^{\top}\left(\boldsymbol{\mu}_{S}(t)-r(t) \mathbf{1}\right)\right)-c_{i}(t)\right) d t+W_{i}(t) \boldsymbol{\pi}_{i}(t)^{\top} \boldsymbol{\sigma}_{S}(t) d \mathbf{Z}(t),\right.
$$

where $\beta$ is the discount rate, $c_{i}(t)$ is the consumption rate, $W_{i}(t)$ is investor $i$ 's wealth and $\boldsymbol{\pi}_{i}(t) \equiv\left(\pi_{1}^{i}(t), \cdots, \pi_{K}^{i}(t)\right)^{\top}$ is the vector of the wealth proportions invested in the stocks. Due to market completeness, (13) is equivalent to the static budget constraint

$$
\mathbb{E}\left[\int_{0}^{\infty} \xi(t) c_{i}(t) d t\right] \leq W_{i}(0)
$$

\footnotetext{
$\overline{13}$ Note that when the bias correlation $\rho=1$ and the absolute bias ratio $\vartheta=1$, the beliefs become homogenous, i.e. $\boldsymbol{\theta}_{A}=\boldsymbol{\theta}_{B}$.
} 
where $W_{i}(0)$ is investor $i$ 's intial wealth. By the first order condition, the optimal consumption of investor $i$ is given by

$$
c_{i}^{*}(t)=\beta W_{i}(0) e^{-\beta t} \frac{M_{i}(t)}{\xi(t)}, \quad i=A, B
$$

The ratio between investors' subjective beliefs can be characterized by

$$
\eta(t) \equiv \frac{M_{A}(t)}{M_{B}(t)}=\exp \left\{-\frac{1}{2}\left(\left\|\boldsymbol{\theta}_{A}\right\|^{2}-\left\|\boldsymbol{\theta}_{B}\right\|^{2}\right) t+\left(\boldsymbol{\theta}_{A}-\boldsymbol{\theta}_{B}\right)^{\top} \mathbf{Z}(t)\right\}
$$

Thus, the relative consumption shares of the two investors are given by

$$
\lambda_{A}(t) \equiv \frac{c_{A}^{*}(t)}{c_{A}^{*}(t)+c_{B}^{*}(t)}=\left(1+\frac{x_{B}}{x_{A}} \eta(t)^{-1}\right)^{-1} \quad \text { and } \quad \lambda_{B}(t)=1-\lambda_{A}(t),
$$

where

$$
x_{A}=\frac{W_{A}(0)}{W_{A}(0)+W_{B}(0)}, \quad \text { and } \quad x_{B}=1-x_{A} .
$$

Now we introduce the index investor $I$ who simply holds the market portfolio and consumes a fixed proportion of the aggregate dividend. The index investor does not affect the equilibrium prices and his consumption is given by

$$
c_{I}^{*}(t)=\frac{W_{I}(0)}{W_{M}(0)} D(t)
$$

where $W_{I}(0)$ is his initial wealth and $W_{M}(0)=W_{A}(0)+W_{B}(0)+W_{I}(0)$ is the total initial market wealth. Note that under logarithmic utility, the index portfolio is optimal under the consensus probability belief (see, for example, Jouini and Napp (2007)),

$$
M(t)=x_{A} M_{A}(t)+x_{B} M_{B}(t)
$$

which satisfies

$$
d M(t)=M(t)\left[\lambda_{A}(t) \boldsymbol{\theta}_{A}+\lambda_{B}(t) \boldsymbol{\theta}_{B}\right]^{\top} d \mathbf{Z}(t)
$$

However, as shown in Jouini and Napp (2007), the index portfolio is no longer optimal when investors have CRRA utility with relative risk aversion coefficient different from one. Lastly, from the market clearing condition, $\sum_{i \in\{A, B, I\}} c_{i}^{*}(t)=D(t)$, the state price density is given by

$$
\xi(t)=M(t) e^{-\beta t} \frac{D(0)}{D(t)} .
$$


Therefore, the security prices are completely determined by investors $A$ and $B$ and the index investor does not play a role.

2.3. Measuring Welfare. Following Fedyk et al. (2013), we measure welfare using the objective expected utilities, i.e. the welfare is measured based on the average realized utilities rather than those under investors' subjective (biased) beliefs.

Definition 2.3. The welfare $\mathcal{U}_{i}$ of investor $i \in\{A, B, I\}$ is given by

$$
\mathcal{U}_{i}=\mathbb{E}_{0}\left[\int_{0}^{\infty} e^{-\beta t}\left(\ln \left(\frac{c_{i}^{*}(t)}{W_{i}(0)}\right)\right) d t\right]
$$

We acknowledge that defining a welfare criterion in an economy with heterogeneous beliefs is not a trivial task. The first problem is whether one should use ex-ante or ex-post measure of the welfare. "People's ex ante welfare is their expected welfare, given their probability judgements, before their expectations are realized. People's ex post welfare is their welfare as things in fact turn out" (Hausman and McPherson 1994, p.396). Starr (1973) argues that we should really care about whether an allocation is ex-post Pareto optimal "that there be redistribution that will increase some trader's realized utility while decreasing no trader's realized utility" (p.82). This is a very strong notion of ex-post optimum; "even if the state of the world were known in the first period, it would be impossible to find a Pareto-superior allocation" (Hammond 1981, p.236). Moreover, the ex-ante and ex-post optima need not be consistent when the subjective beliefs are heterogeneous, marginal rates of substitution that are the same ex-ante may be different ex-post. Harris (1978) proposes to correct for the divergence among the ex-post marginal rates of substitution by quoting ex-ante different set of prices to each individual. One critique of the ex-post approach is that an ex-post optimal allocation can make individuals feel worse off exante. In reality, individuals most likely care about both expected and realized utilities (Barberis and Xiong 2012), they may also deviate from the objectively optimal strategy if they can derive more satisfaction by having an over-optimistic belief ex-ante (Brunnermeier and Parker 2005).

The second problem, as Brunnermeier, Simsek and Xiong (2014) correctly point out, "In many realistic situations, the planner does not observe the objective belief and faces the same difficulty as individuals do in discriminating different beliefs based on available data." To tackle this issue, they introduce a belief neutral welfare criterion, which does not require the planner to fixate on one particular belief as the correct one. According to their welfare criterion, one 
allocation is Pareto inefficient (efficient) if for every reasonable probability measure it is (not) dominated by another allocation. Of course, there will be many scenarios where an allocation is neither Pareto efficient nor inefficient according to the belief-neutral criterion, which makes it difficult for a social planner to determine an optimal allocation.

In this paper we follow Fedyk et al. (2013) to measure the welfare using the expected ex-post utility under the objective probabilities, which in practice can be very difficult to estimate. The social planner, who is concerned about the ex-post welfare of the investors, can either take a stand on what exactly is the objective probability or entertain all combinations of investors' subjective beliefs as reasonable candidates (as in Brunnermeier et al. (2014)). We adopt the former approach, which helps us to clearly identify situations in which the active investing becomes harmful to the investors and switching to the index portfolio can significantly improve their expected ex-post welfare. As our welfare analysis will show, there are cases where the welfare cost of the active investing is almost comparable to "drinking a fatal poison in the mistaken belief that it was water" (Hausman and McPherson 1994, p.396). The drawback of our approach is that it forces the planner to fixate on one probability belief as the truth, also any policies that the planner makes will inevitably make the investors feel worse off ex-ante though they are expected to be better off ex-post.

2.4. Welfare Analysis. According to Definition 2.3, the difference in the expected ex-post welfare between investors $A$ and $B$ is given by

$$
\begin{aligned}
\mathcal{U}_{A}-\mathcal{U}_{B} & =\mathbb{E}_{0}\left[\int_{0}^{\infty} e^{-\beta t} \ln \left(\frac{c_{A}^{*}(t) / W_{A}(0)}{c_{B}^{*}(t) / W_{B}(0)}\right) d t\right] \\
& =\mathbb{E}_{0}\left[\int_{0}^{\infty} e^{-\beta t} \ln (\eta(t)) d t\right]=\int_{0}^{\infty} e^{-\beta t} \frac{1}{2}\left(\left\|\boldsymbol{\theta}_{B}\right\|^{2}-\left\|\boldsymbol{\theta}_{A}\right\|^{2}\right) t d t \\
& =\frac{1}{2 \beta^{2}}\left(\left\|\boldsymbol{\theta}_{B}\right\|^{2}-\left\|\boldsymbol{\theta}_{A}\right\|^{2}\right)>0
\end{aligned}
$$

which is not surprising since investor $A$ has a more accurate belief than investor $B$. However, the comparison between investor $A$ and the index investor $I$ is more complex. We show that, although investor $A$ has a higher consumption growth than $I$ in the long run, i.e.,

$$
\lim _{t \rightarrow \infty} \ln \left(\frac{c_{A}^{*}(t) / W_{A}(0)}{c_{I}^{*}(t) / W_{I}(0)}\right)=\ln \left(1+\frac{x_{B}}{x_{A}}\right)>0,
$$


this does not guarantee that $\mathcal{U}_{I}<\mathcal{U}_{A}$. The difference in the welfare between $A$ and $I$ is characterized by the following lemma.

Lemma 2.4. The welfare of the index investor relative to that of investor $A$ is given by

$$
\mathcal{U}_{I}-\mathcal{U}_{A}=\mathbb{E}_{0}\left[\int_{0}^{\infty} e^{-\beta t} \ln \left(x_{A}+x_{B} \eta(t)^{-1}\right) d t\right]
$$

where $\eta(t)$ is given by Equation (16).

Based on Lemma 2.4, we now present the main result for the baseline model under logarithmic preference.

Proposition 2.5. Assume the investors have logarithmic utility,

- if $\rho>0$ and

$$
(S 1): \vartheta \leq \rho,
$$

then $\mathcal{U}_{I}<\mathcal{U}_{A}$. Conversely, if $\mathcal{U}_{A}<\mathcal{U}_{I}$, then $\rho<\vartheta \leq 1$.

- if $\rho<1, x_{A}=x_{B}$ and

$$
(S 2): \vartheta=1
$$

then $\mathcal{U}_{A}<\mathcal{U}_{I}$.

Proposition 2.5 shows that, (i) when the bias correlation is sufficiently high, the relatively accurate investor $(A)$ achieves a better welfare than the index investor; (ii) when both investors are equally biased, the index investor always achieves a better welfare. The intuition is the following. When the bias correlation $\rho=1$, the investors make the same mistakes about the drifts, that is, they are either both optimistic or both pessimistic. In this case, the consensus belief is always more (less) accurate than investor $B(A)$ 's belief. Since the index portfolio is optimal for a log utility maximizer endowed with the consensus belief, it achieves a better (worse) welfare than investor $B(A)$. In contrast, when $\rho<1$, the consensus belief is able to diversify away some of the biases of the investor. Therefore it becomes possible for the index investor to achieve a better welfare than both the active investors depending on the absolute bias ratio $\vartheta$. In the case where $\vartheta=1$, the index investor always achieves a better welfare.

One may find it counter-intuitive that although investor $A$ has the highest consumption growth in the long-run, he does not necessarily achieve a better welfare than the index portfolio. The explanation is the following. Investor $A$ trades speculatively due to his disagreement with investor 


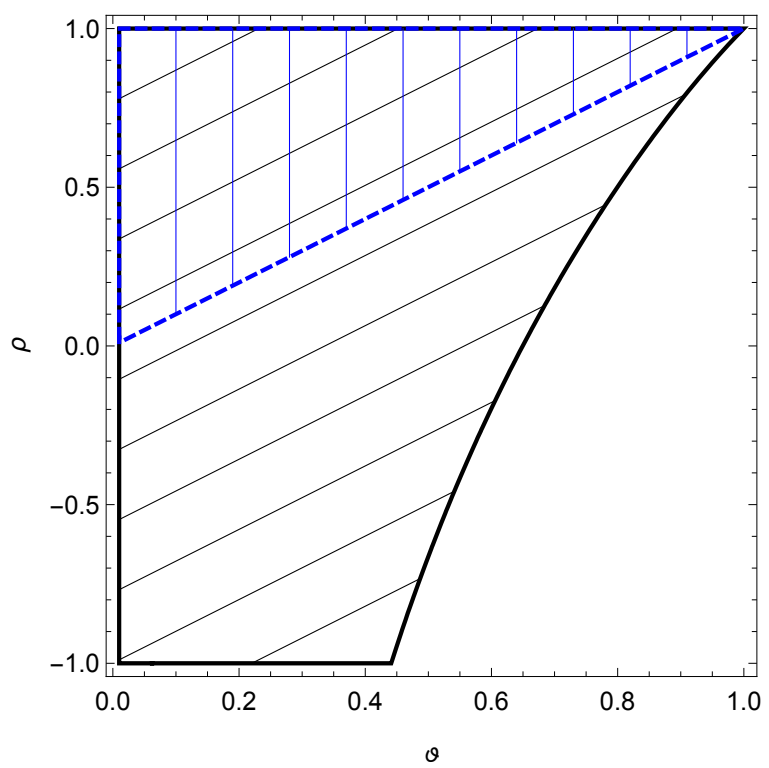

FIGURE 1. Investor $A$ 's outperforming region $\Omega_{A}$ (marked by $45^{\circ}$ lines with a solid boundary) and sufficient region $\Omega_{A}^{S}$ (marked by vertical lines with a dashed boundary).

$B$, but also trades for risk-sharing. When investor $A$ has the correct belief, his consumption plan finds the optimal balance between speculation and risk-sharing, which maximizes his welfare under the objective probabilities. However, when his belief is incorrect, such balance is distorted and he may over-speculate, which makes his consumption path too volatile.

2.5. Numerical Analysis. Proposition 2.5 only provides sufficient conditions for $\mathcal{U}_{I}<\mathcal{U}_{A}$ and $\mathcal{U}_{A}<\mathcal{U}_{I}$. To have a better understanding about the relationship between the absolute bias ratio, the bias correlation and the welfare of the active and index investors, we conduct a numerical analysis to examine the outperforming region $\Omega_{A}$, where investor $A$ achieves a better welfare than the index investor $I$, that is, $\mathcal{U}_{I}<\mathcal{U}_{A}$. Therefore, outside the region $\Omega_{A}$, the index investor outperforms investor $A$, i.e., $\mathcal{U}_{A}<\mathcal{U}_{I}$. We compare $\Omega_{A}$ to the sufficient region $\Omega_{A}^{S}$ defined by condition (S1) in Proposition 2.5, obviously $\Omega_{A}^{S} \subset \Omega_{A}$.

In Figure 1 we set investor $B$ 's absolute bias $\left\|\boldsymbol{\theta}_{B}\right\|=0.25$ and consider the absolute bias ratio $0<\vartheta \leq 1$. Figure 1 leads to three observations. (i) We observe that both the outperforming and sufficient regions $\Omega_{A}$ and $\Omega_{A}^{S}$ are increasing with the bias correlation $\rho$. Therefore, $A$ is more likely to outperform $I$ when $\rho \rightarrow 1$. On the other hand, when $\rho \rightarrow-1, I$ is more likely to outperform $A$. (ii) When $\rho<0$, the sufficient region $\Omega_{A}^{S}$ is empty, but not the outperforming region $\Omega_{A}$. Therefore, when the belief biases are negatively correlated, the index investor does 
not always outperform investor $A$, depending on the absolute bias ratio $\vartheta$. (iii) When $\vartheta=1$ and $\rho<1$, the index investor always outperforms investor $A$, which is consistent with (S2).

The above analysis shows that an indexing portfolio can potentially provide better expected ex-post welfare than all active portfolios. We now conduct a welfare analysis to quantitatively measure the potential improvement in the welfare when investor $A$ switches to the index portfolio, especially when the biases are of similar magnitude and are negatively correlated among the investors. More specifically, we use numerical integration to compute the difference in welfare between $A$ and $I$.

From Definition 2.3, the expected ex-post welfare of one unit of wealth invested in the index portfolio is given by

$$
\mathcal{U}_{I}=\mathbb{E}_{0}\left[\int_{0}^{\infty} e^{-\beta t} \ln \left(\beta \frac{D(t)}{D(0)}\right) d t\right] .
$$

In order to evaluate (23) in closed form, we assume only the first stock is in a positive supply, that is $N_{1}=1$ and $N_{k}=0, k=2, \cdots, K$. The mean growth rate and volatility of the aggregate dividend process are given by $\mu=\mu_{1}$ and $\sigma=\sigma_{1}$ respectively. Thus, we have from (23) that

$$
\mathcal{U}_{I}=\frac{1}{\beta} \ln (\beta)+\frac{1}{\beta^{2}}\left(\mu-\sigma^{2} / 2\right) .
$$

Furthermore, we use numerical integration to evaluate $\mathcal{U}_{I}-\mathcal{U}_{A}$ in equation (22) assuming $x_{A}=$ $x_{B}=\frac{1}{2}$.

Table 1 reports ${ }^{14}\left(\mathcal{U}_{I}-\mathcal{U}_{A}\right) /\left|\mathcal{U}_{A}\right|$ for different levels of the bias correlation $\rho$ and the absolute bias ratio $\vartheta$ when investor $B$ 's absolute bias is relatively small in panel A and large in panel B. The results show that switching to an index portfolio can improve the expected the ex-post welfare of investor $A$ significantly, especially when $\rho<0$ and $\vartheta \rightarrow 1$. For example, in the case where $\rho=-0.8$ and $\vartheta=1$, the welfare improvement for investor $A$ is $69.8 \%$ when $\left\|\boldsymbol{\theta}_{B}\right\|=1$ and $20 \%$ when $\left\|\boldsymbol{\theta}_{B}\right\|=0.25$. However, the amount of improvement decreases with the bias correlation. For example, when $\rho=0.8$ and $\vartheta=1$, the improvement reduces to $39.2 \%$ when $\left\|\boldsymbol{\theta}_{B}\right\|=1$ and $4.2 \%$ when $\left\|\boldsymbol{\theta}_{B}\right\|=0.25$. Moreover, the results show that it is relatively more difficult for the index portfolio to provide welfare improvement when the biases are more positively correlated and the investor with the relatively less accurate belief has a larger absolute bias. Overall, under logarithmic utility, we need $\vartheta \geq 0.6$ in order for $\left(\mathcal{U}_{I}-\mathcal{U}_{A}\right) /\left|\mathcal{U}_{A}\right|>0$ when

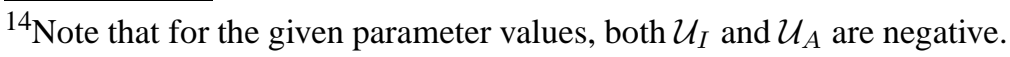




\begin{tabular}{ccccccc}
\multicolumn{7}{c}{ Panel A: $\left\|\boldsymbol{\theta}_{B}\right\|=0.25$} \\
\hline$\vartheta$ & 0.01 & 0.2 & 0.4 & 0.6 & 0.8 & 1.0 \\
\hline$\rho=0.8$ & -0.090 & -0.102 & -0.101 & -0.083 & -0.040 & 0.042 \\
$\rho=0.5$ & -0.089 & -0.094 & -0.084 & -0.055 & 0.000 & 0.087 \\
$\rho=0.0$ & -0.089 & -0.081 & -0.057 & -0.014 & 0.052 & 0.140 \\
$\rho=-0.5$ & -0.088 & -0.069 & -0.033 & 0.020 & 0.092 & 0.180 \\
$\rho=-0.8$ & -0.088 & -0.062 & -0.020 & 0.039 & 0.113 & 0.200 \\
\hline \multicolumn{7}{c}{ Panel B: $\left\|\boldsymbol{\theta}_{B}\right\|=1.0$} \\
\hline$\vartheta$ & 0.01 & 0.2 & 0.4 & 0.6 & 0.8 & 1.0 \\
\hline$\rho=0.8$ & -0.331 & -0.335 & -0.335 & -0.329 & -0.290 & 0.392 \\
$\rho=0.5$ & -0.331 & -0.332 & -0.328 & -0.309 & -0.200 & 0.529 \\
$\rho=0.0$ & -0.330 & -0.327 & -0.313 & -0.262 & -0.032 & 0.625 \\
$\rho=-0.5$ & -0.330 & -0.321 & -0.293 & -0.205 & 0.110 & 0.677 \\
$\rho=-0.8$ & -0.330 & -0.316 & -0.280 & -0.167 & 0.180 & 0.698 \\
\hline
\end{tabular}

TABLE 1. The improvement in the welfare $\left(\mathcal{U}_{I}-\mathcal{U}_{A}\right) /\left|\mathcal{U}_{A}\right|$ by switching to an index portfolio, for absolute bias ratio $\vartheta \in[0.01,1.0]$, bias correlation $\rho \in$ $[-0.8,0.8]$, subjective discount rate $\beta=0.02$, and the mean growth rate and volatility of the aggregate dividend process are given by $\mu=0.02$ and $\sigma=0.02$ respectively.

$\left\|\boldsymbol{\theta}_{B}\right\|=0.25$ (panel A), and $\vartheta \geq 0.8$ when $\left\|\boldsymbol{\theta}_{B}\right\|=1$ (panel B). Therefore, the absolute bias ratio plays a more important role than the bias correlation.

In summary, we have shown that, under logarithmic utility and constant beliefs, the index investor is more likely to achieve a better welfare than the active investors when (i) the active investors' are close to being equally biased and (ii) their biases are more negatively correlated, leading to a diversification effect on the consensus belief. Intuitively, a negative (positive) bias correlation amplifies (mitigates) the negative effect of the belief bias of the active investors on their welfare.

2.6. Performance Analysis. As we claimed earlier, the relatively more accurate investor $(A)$ will drive the less accurate investor $(B)$ out of the market in the long run. In the case of constant beliefs, we can compute this market-selection time explicitly as follows.

Lemma 2.6. Define a market-selection time $\tau>0$ as the expected first time the consumption ratio between $A$ and $B$ reaches $l \leq x_{A} / x_{B}$, i.e.

$$
\tau \equiv \mathbb{E}_{0}\left[\inf \left\{t: c_{A}^{*}(t) / c_{B}^{*}(t)=l\right\}\right]
$$


Then, the market-selection time is given by

$$
\tau=\left(\frac{1}{2}\left(\left\|\boldsymbol{\theta}_{B}\right\|^{2}-\left\|\boldsymbol{\theta}_{A}\right\|^{2}\right)\right)^{-1}\left(\ln (l)-\ln \left(x_{A} / x_{B}\right)\right) .
$$

Lemma 2.6 shows that the market selection time depends only on the absolute belief biases and not on the bias correlation. Therefore, a fast market selection does not necessarily imply that the active investing is better off than the index investing or vice versa. To illustrate, we set the absolute bias to be relative large for agent $B\left(\left\|\boldsymbol{\theta}_{B}\right\|=1\right)$ in Figure 2 and compute the market-selection time for different absolute bias ratios. It can be seen that even for $\vartheta=0.8$, it takes less than 10 years on average for the consumption ratio to reach $l=5$. However, as we have shown in Table 1 panel B, the welfare comparison between the active and index investing depends crucially on the bias correlation (e.g $\left(\mathcal{U}_{I}-\mathcal{U}_{A}\right) /\left|\mathcal{U}_{A}\right|>(<) 0$ when $\rho=-0.8(0.8)$ ). Intuitively, a negative bias correlation makes the market selection process more volatile, which is detrimental to investor $A$ 's expected ex-post welfare.

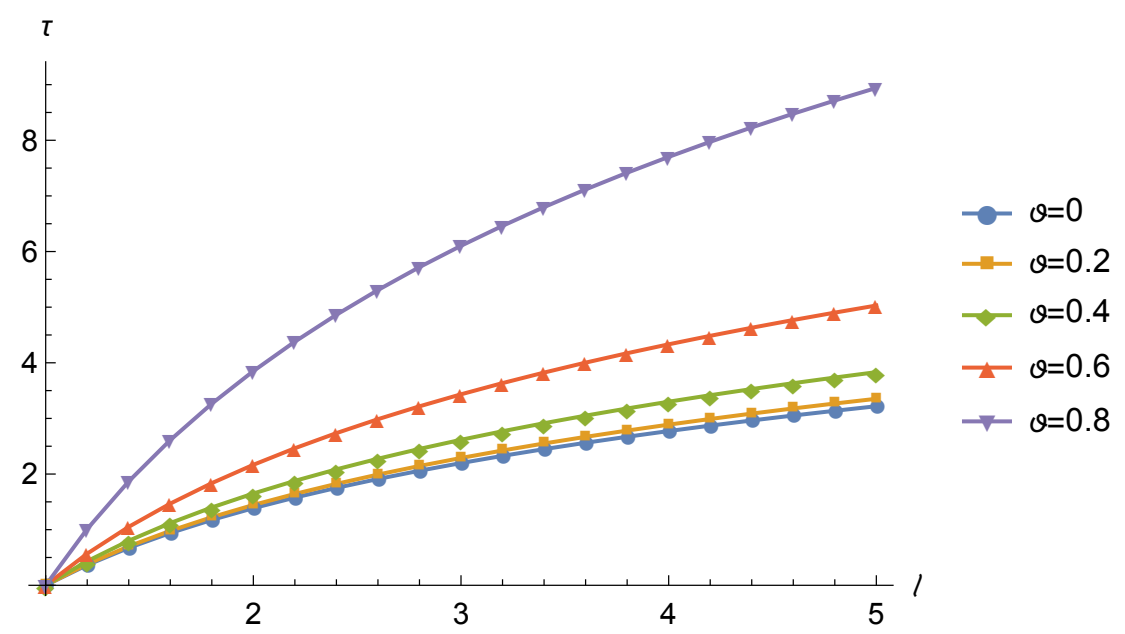

FIGURE 2. Market selection time $\tau=\mathbb{E}\left[\inf \left\{t: c_{A}^{*}(t) / c_{B}^{*}(t)=l\right\}\right]$ under constant beliefs. Investor $B$ 's absolute belief bias $\left\|\boldsymbol{\theta}_{B}\right\|=1$, initial consumption ratio $x_{A} / x_{B}=1$.

We can also compute the Sharpe ratios for the active and index portfolios, which are defined under the objective probability measure $\mathcal{P}$ as

$$
\mathcal{S}_{i}(t) \equiv \frac{\mathbb{E}_{t}\left[d R_{p}^{i}(t)-r(t) d t\right]}{\sqrt{\operatorname{Var}_{t}\left[d R_{p}^{i}(t)\right]}}
$$

for $i \in\{A, B, I\}$, where

$$
d R_{p}^{i}(t)=r(t) d t+\boldsymbol{\pi}_{i}^{*}(t)^{\top}\left(\boldsymbol{\mu}_{S}(t)-r(t) \mathbf{1}\right) d t+\boldsymbol{\pi}_{i}^{*}(t)^{\top} \boldsymbol{\sigma}_{S}(t) d \mathbf{Z}(t)
$$


is portfolio $i$ 's instantaneous return. In the case of logarithmic utility, we can express the portfolio return in terms of the changes in consumption, i.e.,

$$
d R_{p}^{i}(t)=\frac{d W_{i}(t)}{W_{i}(t)}+\frac{c_{i}^{*}(t)}{W_{i}(t)} d t=\frac{d c_{i}^{*}(t)}{c_{i}^{*}(t)}+\beta d t
$$

For simplicity, we assume only the first risky asset is in a positive supply and agents agree on the drift of the aggregate dividend process, that is $\mu_{1}^{i}=\mu$ for $i=A, B$. In this case, the risk-free rate is given by $r=\beta+\mu-\sigma^{2}$ and the Sharpe ratio of the index portfolio is constant,

$$
\mathcal{S}_{I}(t)=\frac{\mu+\beta-r}{\sigma}=\sigma
$$

In comparison, the Sharpe ratios of the active portfolios depends on the distribution of the consumption shares between investors $A$ and $B$, i.e.,

$$
\begin{aligned}
& \mathcal{S}_{A}(t)=\frac{\sigma^{2}+\frac{1}{2} \lambda_{B}(t)\left(\left\|\boldsymbol{\theta}_{B}\right\|^{2}-\left\|\boldsymbol{\theta}_{A}\right\|^{2}\right)+\lambda_{B}(t)^{2}\left\|\boldsymbol{\theta}_{A}-\boldsymbol{\theta}_{B}\right\|^{2}}{\sqrt{\sigma^{2}+\lambda_{B}(t)^{2}\left\|\boldsymbol{\theta}_{A}-\boldsymbol{\theta}_{B}\right\|^{2}}} \\
& \mathcal{S}_{B}(t)=\frac{\sigma^{2}-\frac{1}{2} \lambda_{A}(t)\left(\left\|\boldsymbol{\theta}_{B}\right\|^{2}-\left\|\boldsymbol{\theta}_{A}\right\|^{2}\right)+\lambda_{A}(t)^{2}\left\|\boldsymbol{\theta}_{A}-\boldsymbol{\theta}_{B}\right\|^{2}}{\sqrt{\sigma^{2}+\lambda_{A}(t)^{2}\left\|\boldsymbol{\theta}_{A}-\boldsymbol{\theta}_{B}\right\|^{2}}} .
\end{aligned}
$$

Equations (30), (31) and (32) lead to the following observations. First, since $\left\|\boldsymbol{\theta}_{A}\right\| \leq\left\|\boldsymbol{\theta}_{B}\right\|$ investor $A$ always has the highest Sharpe ratio regardless of the distribution of the consumption shares. Second, when investors $A$ and $B$ have the same absolute belief bias, they both outperform the index portfolio, that is $\mathcal{S}_{A}=\mathcal{S}_{B} \geq \mathcal{S}_{I}$. These results are in sharp contrast to the welfare analysis. This illustrates that the Sharpe ratio can be misleading in favoring active investing over the index portfolio even when active investing has a significant welfare cost. Furthermore, Figure 3 (a) and (b) show that $\mathcal{S}_{A} \downarrow$ and $\mathcal{S}_{B} \uparrow$ when $\vartheta \rightarrow 1$. When $\vartheta=1$ both the active portfolios outperform the index portfolio. More interestingly, Figure 3 (c) and (d) show that $\mathcal{S}_{A} \uparrow$ and $\mathcal{S}_{B} \uparrow$ when $\rho \rightarrow-1$, which is again the opposite to what we found from the welfare results in Table 1 .

Our intuition for the above results is as follows. The Sharpe ratio only considers the trade-off between standard deviation and expected excess return. In this scenario the active portfolios actually improve the trade-off by taking on more risk. In comparison, the welfare analysis cares about whether any increase in the volatility of the consumption growth results positive growth in the consumption share. Therefore, the two performance measures are not consistent in general. 


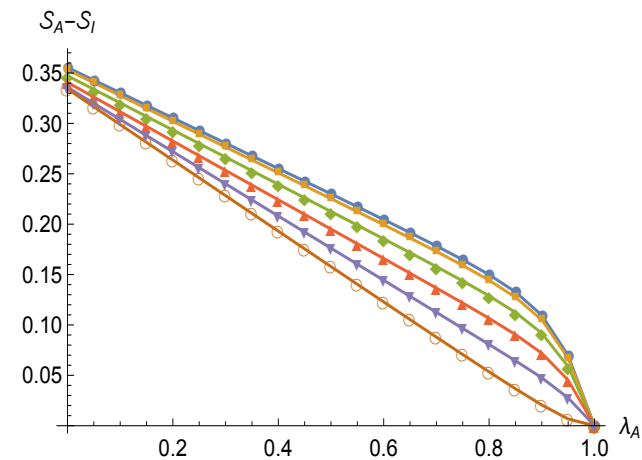

(a) $\mathcal{S}_{A}-\mathcal{S}_{I} ; \rho=0$

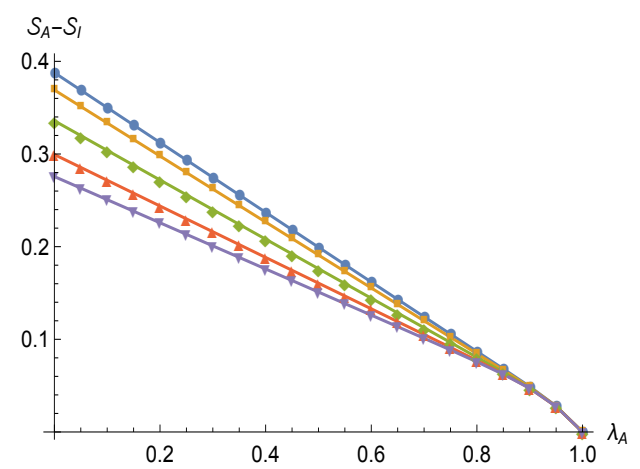

(c) $\mathcal{S}_{A}-\mathcal{S}_{I} ; \vartheta=0.8$
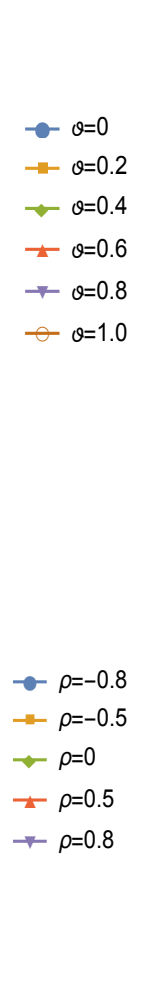

FIGURE 3. Excess Sharpe ratio for active portfolios of investors $A$ and $B$ for bias correlation $\rho=0$ and absolute bias ratio $\vartheta \in[0,1]$ in plots (a) and (b), $\vartheta=0.8$ and $\rho \in[-0.8,0.8]$ in plots (c) and (d). Investor $B$ 's absolute bias is set to $\left\|\boldsymbol{\theta}_{B}\right\|=0.25$ and volatility of aggregate consumption growth $\sigma=0.02$.

\section{CRRA UTILITY}

We extend the previous analysis to CRRA utility with a constant relative risk aversion $\gamma>0$. Investors $A$ and $B$ maximize

$$
\mathbb{E}_{0}\left[\int_{0}^{T} e^{-\beta t} M_{i}(t) \frac{c_{i}(t)^{1-\gamma}}{1-\gamma} d t\right], \quad i \in\{A, B\},
$$

subject to the budget constraint (14). Following Jouini and Napp (2007), the optimal consumption of investor $i$ is given by

$$
c_{i}^{*}(t)=\left(\frac{e^{-\beta t} M_{i}(t)}{y_{i} \xi(t)}\right)^{\frac{1}{\gamma}}
$$

where $y_{i}$ is the Lagrange multiplier corresponding to the budget constraint. The initial wealth of investor $i$ is given by

$$
W_{i}(0)=\mathbb{E}_{0}\left[\int_{0}^{\infty}\left(\frac{e^{-\beta t} M_{i}(t)}{y_{i}}\right)^{\frac{1}{\gamma}} \xi(t)^{\frac{\gamma-1}{\gamma}} d t\right] .
$$


Furthermore, the optimal consumption of the index portfolio remains the same as that in (18). From the market clearing condition $\sum_{i \in\{A, B, I\}} c_{i}^{*}(t)=D(t)$, we obtain the state price density process,

$$
\xi(t)=e^{-\beta t} M(t)\left(\frac{D(t)}{D(0)}\right)^{-\gamma},
$$

where the consensus characteristic

$$
M(t)=\left(x_{A} M_{A}(t)^{\frac{1}{\gamma}}+x_{B} M_{B}(t)^{\frac{1}{\gamma}}\right)^{\gamma}
$$

and 15

$$
x_{i}=\frac{c_{i}^{*}(0)}{D(0)} \frac{W_{M}(0)}{W_{A}(0)+W_{B}(0)}, \quad i=A, B .
$$

Therefore, given (18), (33) and (35), the expected ex-post instantaneous utility of investor $A$ can be written as the following 10 ,

$$
\begin{aligned}
& \frac{1}{1-\gamma} \mathbb{E}_{0}\left[\left(\frac{c_{A}^{*}(t)}{W_{A}(0)}\right)^{1-\gamma}\right] \\
& =\frac{1}{1-\gamma}\left(\frac{c_{A}^{*}(0)}{W_{A}(0)} \frac{W_{M}(0)}{D(0)}\right)^{1-\gamma} \mathbb{E}_{0}\left[\left(x_{A}+x_{B} \eta(t)^{-\frac{1}{\gamma}}\right)^{\gamma-1}\left(\frac{c_{I}^{*}(t)}{W_{I}(0)}\right)^{1-\gamma}\right] .
\end{aligned}
$$

Equation (38) shows that, unlike the case of logarithmic utility, the comparison between investor $A$ and the index portfolio $I$ depends on the distributions of the initial wealth and consumption among the investors. In the following lemma, we derive a condition under which we can express $\frac{1}{1-\gamma} \mathbb{E}_{0}\left[\left(\frac{c_{A}^{*}(t)}{W_{A}(0)}\right)^{1-\gamma}\right]$ in terms of $\frac{1}{1-\gamma} \mathbb{E}_{0}\left[\left(\frac{c_{I}^{*}(t)}{W_{I}(0)}\right)^{1-\gamma}\right]$ in a closed form that is independent of the initial wealth and consumption distributions. This helps analytical tractability for the welfare analysis.

Lemma 3.1. Assume (i) asset 1 is in a positive supply of one share $\left(N_{1}=1\right)$ and all other assets are in zero net supply $\left(N_{k}=0, k=2, \cdots, K\right)$, (ii) investors $A$ and $B$ have the same initial wealth, that is $W_{A}(0)=W_{B}(0)$, and (iii) investors $A$ and $B$ agree on the drift of the aggregate dividend process, that is, $\theta_{1}^{A}=\theta_{1}^{B}=\theta_{1}$. Then, investors $A$ and $B$ have the same initial consumption, that is $\lambda_{A}(0)=\lambda_{B}(0)$, and the expected ex-post instantaneous utility of

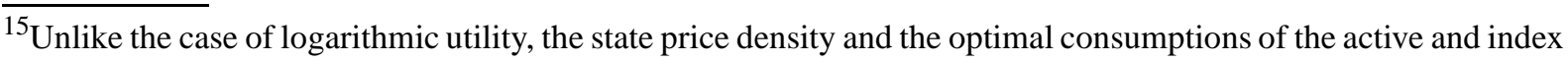
portfolios depend on the distributions of the initial wealth and consumption, though the equilibrium price is only determined by the beliefs of investors $A$ and $B$. Note that $x_{A}+x_{B}=\frac{\left(c_{A}(0)+c_{B}(0)\right) / D(0)}{\left(W_{A}(0)+W_{B}(0)\right) / W_{M}(0)}=\frac{1-c_{I}(0) / D(0)}{1-W_{I}(0) / W_{M}(0)}$ and $c_{I}(0) / D(0)=W_{I}(0) / W_{M}(0)$. Hence $x_{A}+x_{B}=1$.

${ }^{16}$ The expected ex-post instantaneous utility of investor $B$ can be written in a similar way.
} 
investor $A$ is given by

$$
\frac{1}{1-\gamma} \mathbb{E}_{0}\left[\left(\frac{c_{A}^{*}(t)}{W_{A}(0)}\right)^{1-\gamma}\right]=\frac{1}{1-\gamma} \mathbb{E}_{0}\left[\left(\frac{1}{2}\left(1+\eta(t)^{-\frac{1}{\gamma}}\right)\right)^{\gamma-1}\right] \mathbb{E}_{0}\left[\left(\frac{c_{I}^{*}(t)}{W_{I}(0)}\right)^{1-\gamma}\right]
$$

3.1. Effect of Risk Aversion. Note that from Lemma 3.1, investor $A$ has a higher expected ex-post instantaneous utility compared to the index portfolio $I$ when

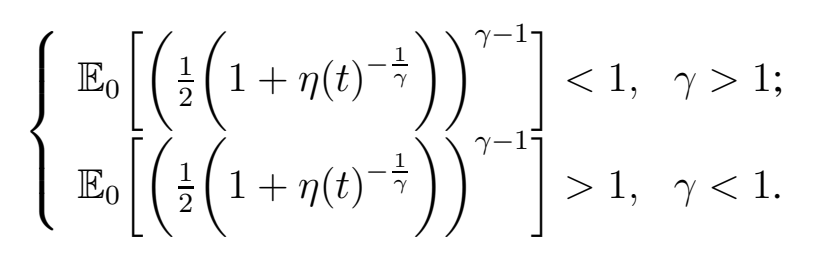

Next we present the main result for the case of CRRA utility.

Proposition 3.2. Under the assumptions of Lemma 3.1 let

$$
\begin{aligned}
& L \equiv \frac{1}{1+\gamma}\left(\rho-\sqrt{\gamma^{2}+\rho^{2}-1}\right) \quad \text { and } \quad R \equiv \frac{1}{1+\gamma}\left(\rho+\sqrt{\gamma^{2}+\rho^{2}-1}\right), \\
& \text { - For } \sqrt{1-\rho^{2}} \leq \gamma<2 \text {, if } \quad(S 3): L \leq \vartheta \leq R,
\end{aligned}
$$

then $\mathcal{U}_{I}<\mathcal{U}_{A}$.

- For $\gamma=2, \mathcal{U}_{I}<\mathcal{U}_{A}$ if and only if

$$
(N S): \vartheta<R
$$

- For $\gamma>2$, if $\mathcal{U}_{I}<\mathcal{U}_{A}$, then

$$
(N): \vartheta<R
$$

Proposition 3.2 provides a sufficient condition (S3) (for $\gamma<2$ ), a necessary and sufficient condition (NS) (for $\gamma=2$ ), and a necessary condition (N) (for $\gamma>2$ ) for investor $A$ to achieve a better welfare than the index investor $I$. It leads to the following four observations. (i) For $\gamma=1$, condition (S3) becomes $0 \leq \vartheta \leq \rho$, which reduces to the sufficient condition (S1) in Proposition 2.5, (ii) When the bias correlation $\rho=1$ and the relative risk aversion coefficient satisfies $1 \leq \gamma<2$, we always have $\mathcal{U}_{I}<\mathcal{U}_{A}$ (since $L \leq 0$ and $R=1$ ). Hence the sufficient condition (S3) is always satisfied. This is consistent with our result in the baseline model. (iii) For $\gamma=2$, the sufficient condition (S3) becomes a necessary and sufficient condition (NS). The lower bound is no longer relevant because $L<0$. Condition (NS) confirms that a more 
negative bias correlation favors the index portfolio. To illustrate, when $\rho=1$, (NS) becomes $\vartheta<1$, which is always true, therefore $\mathcal{U}_{I}<\mathcal{U}_{A}$. However, when $\rho=-1$, (NS) becomes $\vartheta<1 / 3$, hence we have $\mathcal{U}_{I}<\mathcal{U}_{A}$ for $0<\vartheta<1 / 3$ and $\mathcal{U}_{A} \leq \mathcal{U}_{I}$ for $1 / 3 \leq \vartheta \leq 1$. Therefore, the bias correlation makes a significant difference. (iv) For $\gamma>2$, condition (S3) becomes only a necessary condition $(\mathrm{N})$. Note that $R \leq 1$ and $R=1$ if and only if $\rho=1$. When both investors have the absolute bias $(\vartheta=1)$, condition $(\mathrm{N})$ is never satisfied for $\rho<1$. In this case, we have $\mathcal{U}_{A}<\mathcal{U}_{I}$, which is consistent with condition (S2) derived under logarithmic utility.

3.2. Numerical Analysis. To better understand the impact of the risk aversion on the welfare, we conduct a numerical analysis to examine the outperforming region of investor $A$ (in which $\mathcal{U}_{I}<\mathcal{U}_{A}$ ) for risk aversion coefficient $0<\gamma \leq 5$.

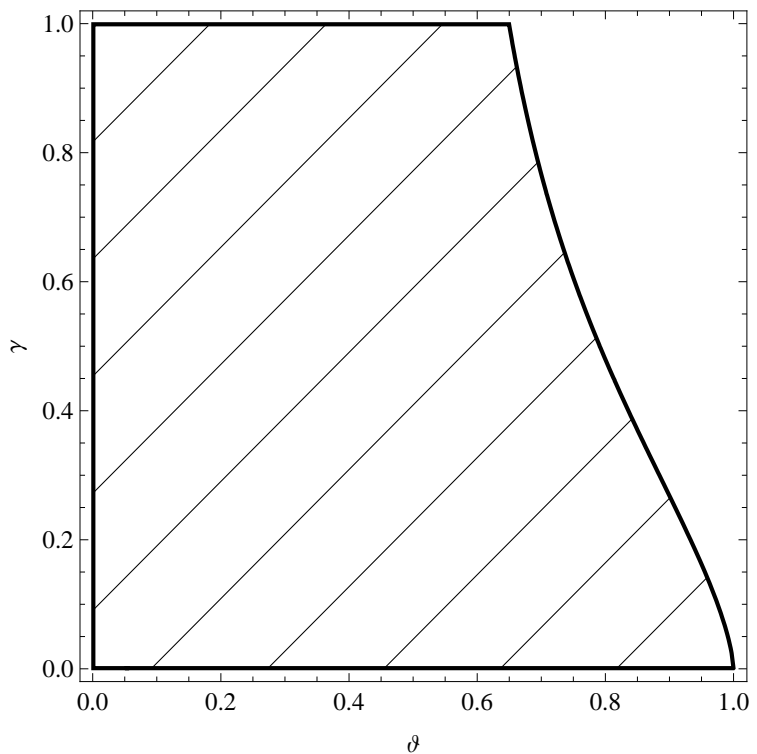

(a) $0<\gamma<1$

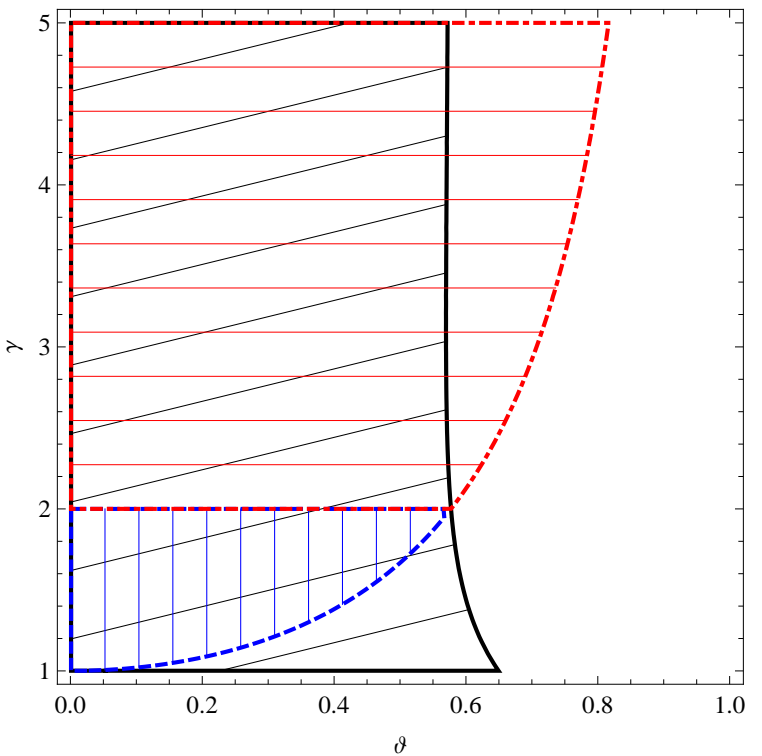

(b) $1<\gamma \leq 5$

FIGURE 4. The outperforming region $\Omega_{A}$ (marked by $45^{\circ}$ lines with a solid boundary), sufficient region $\Omega_{A}^{S}$ (marked by vertical lines with a dashed boundary) and necessary region $\Omega_{A}^{N}$ (marked by horizontal lines with a dot-dashed boundary) for risk aversion $0<\gamma<1$ in plot (a) and $0<\gamma \leq 5$ in plot (b), absolute bias ratio $0 \leq \vartheta \leq 1$, investor $B$ 's absolute bias $\left\|\boldsymbol{\theta}_{B}\right\|=0.25$ and bias correlation $\rho=0$.

In Figure 4, we compare the outperforming region $\Omega_{A}$ with the sufficient region $\Omega_{A}^{S}$ defined by the sufficient condition (S3), and the necessary region $\Omega_{A}^{N}$ defined by the necessary condition (N) in Proposition 3.2. We plot three regions $\Omega_{A}^{S}, \Omega_{A}$ and $\Omega_{A}^{N}$ for the bias correlation $\rho=0$ and the relative risk aversion $0<\gamma<1$ in plot (a) and $1<\gamma \leq 5$ in plot (b). For $\gamma<1$, the sufficient region indicated by condition (S3) is empty $\left(\Omega_{A}^{S}=\emptyset\right)$. In comparison, panel (a) shows that the outperforming region $\Omega_{A}$ covers more than half of the entire space and the 
region is shrinking in $\gamma$. This suggests that, for a given absolute bias ratio, when the biases are uncorrelated, an increase in the risk aversion (up to 1) actually favors the index portfolio. Plot (b) shows that when $\gamma>1$, we have $\Omega_{A}^{S} \subseteq \Omega_{A}$ for $1<\gamma<2, \Omega_{A}^{S}=\Omega_{A}=\Omega_{A}^{N}$ for $\gamma=2$ and $\Omega_{A} \subseteq \Omega_{A}^{N}$ for $2<\gamma<5$. Moreover, as $\gamma$ increases, the outperforming region $\Omega_{A}$ is monotonically decreasing up to approximately $\gamma=2$ and then remains constant, whereas the sufficient/necessary regions $\Omega_{A}^{S}$ and $\Omega_{A}^{N}$ are monotonically increasing.

The above numerical analysis indicates that a higher risk aversion favors the index investor, however the marginal effect is diminishing and disappears after the risk aversion reaches a certain threshold. The intuition is the following. A higher risk aversion leads to increasing concavity in the utility function, which magnifies the negative effect of low consumption shares. However, it also reduces the speculation between the investors, which makes their relative consumption shares less volatile. When the risk aversion is below the threshold, the first effect dominates the second, which makes the active portfolios worse off and favors the index portfolio. On the other hand, when the risk aversion is above the threshold, the two effects offset each other.

To better understand the impact of the bias correlation, we conduct a numerical analysis for $\rho=-1$ and $\rho=1$ respectively. Figure 5 shows the outperforming, sufficient and necessary regions for $\rho=1$ and $0<\gamma<1$ in plot (a), $\rho=1$ and $1<\gamma \leq 5$ in plot (b), $\rho=-1$ and $0<\gamma<1$ in plot (c), and $\rho=-1$ and $1<\gamma \leq 5$ in plot (d). Plots (a) and (b) show that, when the bias correlation $\rho=1$, the outperforming region $\Omega_{A}$ covers the entire space, suggesting that $\mathcal{U}_{I}<\mathcal{U}_{A}$ for all risk aversion $\gamma \in(0,5]$. In contrast, plots (c) and (d) show that when $\rho=-1$, the outperforming region $\Omega_{A}$ becomes significantly smaller compared to the case of $\rho=1$. Therefore, a more negative bias correlation works in favor of the index investor, which is consistent with the intuitions in the baseline model.

We now conduct a welfare analysis to examine the amount of the welfare improvement by switching to an index portfolio for investor $A$. Since the welfare of the index investor $I$ is given by

$$
\begin{aligned}
\mathcal{U}_{I} & =\frac{1}{1-\gamma} \mathbb{E}_{0}\left[\int_{0}^{\infty} e^{-\beta t}\left(\frac{D(t)}{W_{M}(0)}\right)^{1-\gamma} d t\right] \\
& =\left(\frac{W_{M}(0)}{D(0)}\right)^{\gamma-1} \frac{1}{1-\gamma} \int_{0}^{\infty} e^{-\beta t} \mathbb{E}_{0}\left[\left(\frac{D(t)}{D(0)}\right)^{1-\gamma}\right] d t
\end{aligned}
$$




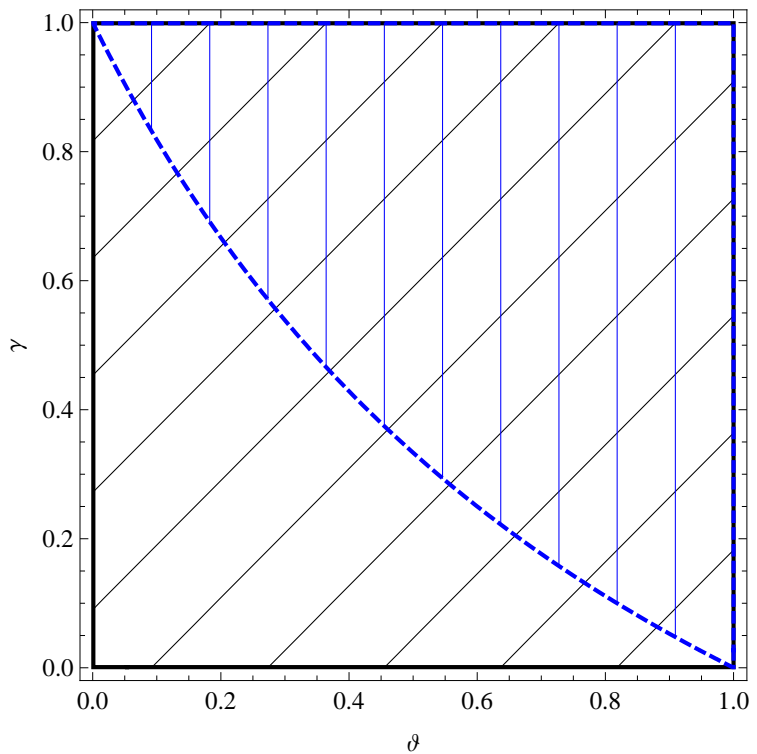

(a) $0<\gamma<1 ; \rho=1$

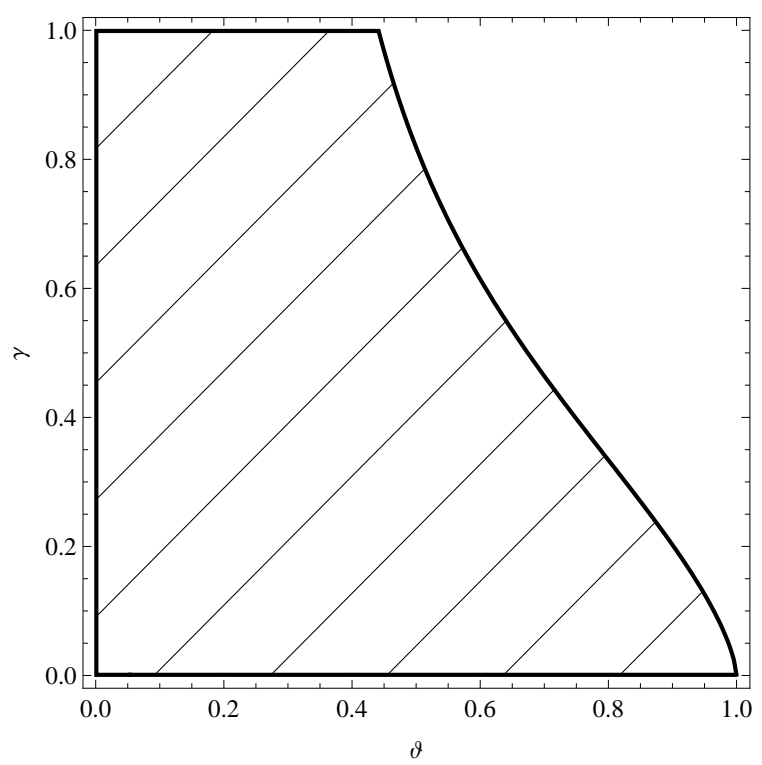

(c) $0<\gamma<1 ; \rho=-1$

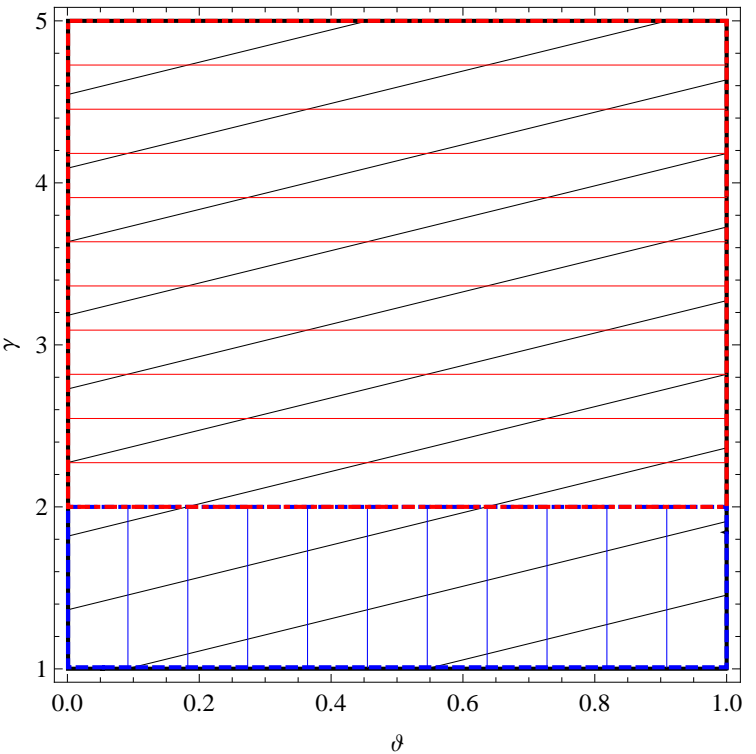

(b) $1<\gamma \leq 5 ; \rho=1$

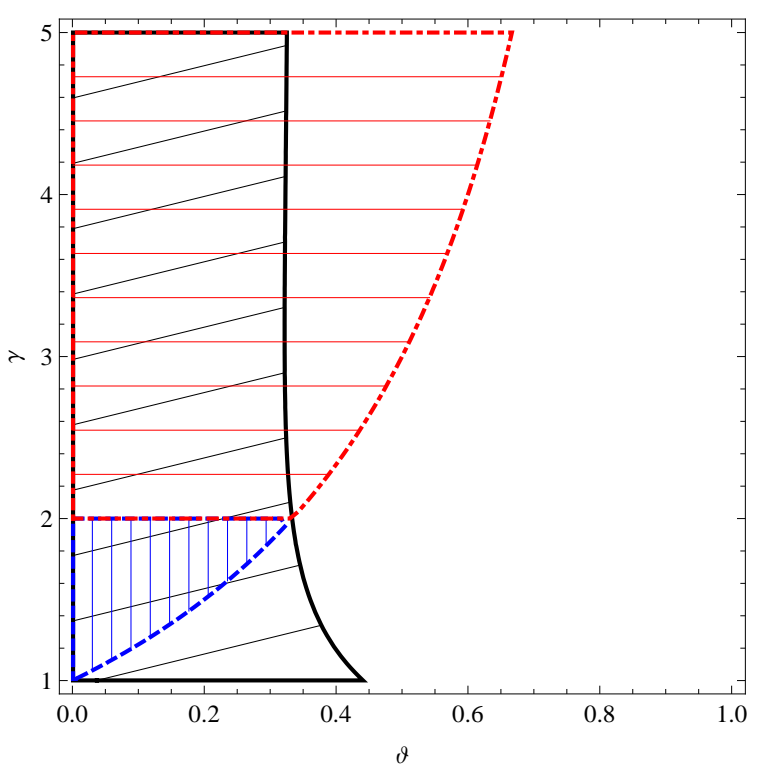

(d) $1<\gamma \leq 5 ; \rho=-1$

FIGURE 5. The outperforming region $\Omega_{A}$ (marked by $45^{\circ}$ lines with a solid boundary), sufficient region $\Omega_{A}^{S}$ (marked by vertical lines with a dashed boundary) and necessary region $\Omega_{A}^{N}$ (marked by horizontal lines with a dot-dashed boundary) for risk aversion $0<\gamma \leq 5$, absolute bias ratio $0 \leq \vartheta \leq 1$, investor $B$ 's absolute belief bias $\left\|\boldsymbol{\theta}_{B}\right\|=0.25$ and bias correlation $\rho=1$ in panels (a) and (b), $\rho=-1$ in panels (c) and (d).

from equation (39), the welfare ratio between portfolios $A$ and $I$ is given by

$$
\frac{\mathcal{U}_{A}}{\mathcal{U}_{I}}=\frac{\int_{0}^{\infty} e^{-\beta t} \mathbb{E}_{0}\left[\left(\frac{1}{2}\left(1+\eta(t)^{-\frac{1}{\gamma}}\right)\right)^{\gamma-1}\right] \mathbb{E}_{0}\left[\left(\frac{D(t)}{D(0)}\right)^{1-\gamma}\right] d t}{\int_{0}^{\infty} e^{-\beta t} \mathbb{E}_{0}\left[\left(\frac{D(t)}{D(0)}\right)^{1-\gamma}\right] d t}
$$

where

$$
\mathbb{E}_{0}\left[(D(t) / D(0))^{1-\gamma}\right]=\exp \left\{\left((1-\gamma)\left(\mu-\gamma \sigma^{2} / 2\right)\right) t\right\}
$$


and

$$
\int_{0}^{\infty} e^{-\beta t} \mathbb{E}_{0}\left[(D(t) / D(0))^{1-\gamma}\right] d t=\beta-(1-\gamma)\left(\mu-\gamma \sigma^{2} / 2\right)
$$

assuming $\beta>(1-\gamma)\left(\mu-\gamma \sigma^{2} / 2\right)$. We use numerical integration to evaluate the welfare in equation (43). Note that for $\gamma>1$, since $\mathcal{U}_{A}<0$ the welfare improvement is given by $\left(\mathcal{U}_{I}-\mathcal{U}_{A}\right) /\left|\mathcal{U}_{A}\right|=1-\mathcal{U}_{I} / \mathcal{U}_{A}$. When $\gamma<1$, since $\mathcal{U}_{A}>0$ we obtain $\left(\mathcal{U}_{I}-\mathcal{U}_{A}\right) /\left|\mathcal{U}_{A}\right|=$ $\mathcal{U}_{I} / \mathcal{U}_{A}-1$

Panel A: $\gamma=1 / 2$

\begin{tabular}{ccccccc}
\hline$\vartheta$ & 0.01 & 0.2 & 0.4 & 0.6 & 0.8 & 1.0 \\
\hline$\rho=0.8$ & -0.191 & -0.204 & -0.205 & -0.188 & -0.123 & 0.102 \\
$\rho=0.5$ & -0.191 & -0.195 & -0.185 & -0.149 & -0.057 & 0.153 \\
$\rho=0.0$ & -0.190 & -0.182 & -0.156 & -0.100 & 0.006 & 0.195 \\
$\rho=-0.5$ & -0.189 & -0.170 & -0.131 & -0.064 & 0.046 & 0.219 \\
$\rho=-0.8$ & -0.189 & -0.163 & -0.118 & -0.047 & 0.064 & 0.229 \\
\hline
\end{tabular}

Panel B: $\gamma=2$

\begin{tabular}{ccccccc}
\hline$\vartheta$ & 0.01 & 0.2 & 0.4 & 0.6 & 0.8 & 1.0 \\
\hline$\rho=0.8$ & -0.091 & -0.106 & -0.103 & -0.080 & -0.034 & 0.041 \\
$\rho=0.5$ & -0.091 & -0.096 & -0.083 & -0.049 & 0.012 & 0.109 \\
$\rho=0.0$ & -0.090 & -0.080 & -0.049 & 0.008 & 0.100 & 0.246 \\
$\rho=-0.5$ & -0.089 & -0.063 & -0.012 & 0.072 & 0.204 & 0.420 \\
$\rho=-0.8$ & -0.088 & -0.052 & 0.012 & 0.114 & 0.277 & 0.551 \\
\hline
\end{tabular}

TABLE 2. The improvement in the expected ex-post welfare $\left(\mathcal{U}_{I}-\mathcal{U}_{A}\right) /\left|\mathcal{U}_{A}\right|$ by switching to an index portfolio under CRRA utility, for absolute bias ratio $\vartheta \in$ $[0.01,1.0]$, bias correlation $\rho \in[-0.8,0.8]$, investor $B$ 's absolute bias $\left\|\boldsymbol{\theta}_{B}\right\|=$ 0.25 , subjective discount rate $\beta=0.02$, and mean growth rate and volatility of the aggregate dividend process are given by $\mu=0.02$ and $\sigma=0.02$ respectively.

Table 2 reports the results for $\left(\mathcal{U}_{I}-\mathcal{U}_{A}\right) /\left|\mathcal{U}_{A}\right|$ for relative risk aversion $\gamma=1 / 2$ and $\gamma=2$. Compared to the results in Table1 1 panel A, there are more (less) cases for $\mathcal{U}_{I}>\mathcal{U}_{A}$ when $\gamma>$ $(<) 1$, which is consistent with the result that a higher risk aversion favors the index portfolio. Panel A shows that, for $\gamma=1 / 2$, welfare improvement does not occur until bias correlation $\rho \leq 0$ and the absolute bias ratio $\vartheta \geq 0$.8. In comparison, panel B shows that, for $\gamma=2$ and $\rho=-0.8$, the welfare improvement occurs even when $\vartheta=0.4$. Again, this is because the effect from increasing concavity in the utility function dominates the effect from decreasing volatility in the consumption shares.

Moreover, the magnitude of the change in the welfare is also much larger compared to the baseline model. For example, when $\rho=-0.8$ and $\vartheta=1$, the improvement in the welfare is $55.1 \%$ for $\gamma=2$ compared to $20 \%$ under logarithmic utility. On the other hand, when 
$\rho=0.8$ and $\vartheta=0.4$, reduction in the welfare is $-20.5 \%$ for $\gamma=1 / 2$ compared to $-10.1 \%$ under logarithmic utility.

\section{IMPACT OF LEARNING}

In this section we relax the assumption of the constant beliefs and assume the investors learn about the true drifts of the dividend processes. To examine the effect of learning, we consider two cases. In the first case, we consider a simple environment where the true drifts are constants as in equation (2). In the second case, we consider a more complex environment where the true drifts follow mean-reverting stochastic processes. In the second case, in addition to the dividend processes, investors continuously observe public signals about the dividends, which they use to update their beliefs. For each dividend process $D_{k}(t)$, let $m_{k}^{i}(t) \equiv \mathbb{E}_{t}^{i}\left[\mu_{k}(t)\right]$ and $\nu_{k}^{i}(t) \equiv \mathbb{E}_{t}^{i}\left[\left(m_{k}^{i}(t)-\mu_{k}(t)\right)^{2}\right]$ be investor $i$ 's subjective belief of its true drift and posterior variance at time $t$ respectively.

4.1. Constant Drifts. Assume that the true drifts of the dividend processes are constant, $\mu_{k}(t)=$ $\mu_{k}$ for $k=1, \cdots, K$ in (2). The investors update their beliefs to learn about the true drifts. The subjective belief of investor $i$ about $\mu_{k}$ evolves according to

$$
d m_{k}^{i}(t)=\frac{\nu_{k}^{i}(t)}{\sigma_{k}^{2}}\left(\frac{d D_{k}(t)}{D_{k}(t)}-m_{k}^{i}(t) d t\right), \quad k=1, \cdots, K, \quad i=A, B
$$

where the posterior variance satisfies the ordinary differential equation

$$
\frac{d \nu_{k}^{i}(t)}{d t}=-\frac{\nu_{k}^{i}(t)^{2}}{\sigma_{k}^{2}}
$$

and the initial condition is characterized by prior distribution $\mu_{k} \sim \mathcal{N}\left(m_{k}^{i}(0), \nu_{k}^{i}(0)\right)$. From (44), the deviation of investor $i$ 's belief from the truth is given by

$$
d \theta_{k}^{i}(t) \equiv d\left(\frac{\mu_{k}^{i}(t)-\mu_{k}}{\sigma_{k}}\right)=\frac{\nu_{k}^{i}(t)}{\sigma_{k}^{2}}\left(d Z_{k}(t)-\theta_{k}^{i}(t) d t\right), \quad k=1, \cdots, K, \quad i=A, B
$$

For simplicity, we assume investor $B$ has constant beliefs $\mu_{k}^{B}$, which is a special case of (45) where the initial posterior variance $\nu_{k}^{B} \rightarrow 0$. Thus investor $B$ 's deviation from the truth is given by $\theta_{k}^{B}=\left(\mu_{k}^{B}-\mu_{k}\right) / \sigma_{k}$ for $k=1, \cdots, K$, meaning that investor $B$ makes persistent mistakes about the true drifts whereas investor $A$ gradually learns about the truth. 
We assume investors $A$ and $B$ have logarithmic utility. The difference in the welfare $\mathcal{U}_{I}-\mathcal{U}_{A}$ can then be computed by Monte Carlo simulation using equation (22) with $x_{A}=x_{B}=\frac{1}{2}$. Moreover, there are two risky assets, which are claims to the dividend processes, however only the first risky asset is in a positive supply, that is $N_{1}=1$ and $N_{2}=0$. In this case, the welfare of the index investor can be computed by (24). Lastly, we assume $\theta_{k}^{A}(0)=\theta_{k}^{B}$ and $\nu_{k}^{A}(0)=\nu$ for $k=1,2$, meaning investors $A$ and $B$ initially have the same beliefs about the drifts. We simulate 10,000 paths of the processes $\left\{\ln \left(M_{i}(t)\right)\right\}_{i \in\{A, B\}}$ under the true probability measure $\mathcal{P}$ using a time increment of $\Delta t=0.01$ for $T=350$ years in order to evaluate (22).

\begin{tabular}{cccccc}
\hline$\nu$ & $0.01^{2}$ & $0.02^{2}$ & $0.04^{2}$ & $0.08^{2}$ & $0.1^{2}$ \\
\hline$\theta_{k}^{B}=-0.25$ & -0.019 & 0.127 & 0.291 & 0.437 & 0.480 \\
$\theta_{k}^{B}=-0.5$ & -0.211 & -0.160 & -0.062 & 0.090 & 0.134 \\
$\theta_{k}^{B}=-1.0$ & -0.281 & -0.279 & -0.260 & -0.215 & -0.192 \\
\hline
\end{tabular}

TABLE 3. Improvement in the welfare $\left(\mathcal{U}_{I}-\mathcal{U}_{A}\right) /\left|\mathcal{U}_{A}\right|$ by switching to an index portfolio under Bayesian learning with constant drifts. Investor $A$ 's initial prior $\mu_{k} \sim \mathcal{N}\left(\theta_{k}^{B}, \nu\right)$, subjective discount rate $\beta=0.02$, drift and volatility for the dividend processes are given by $\mu_{k}=0.02$ and $\sigma_{k}=0.02$ respectively.

Table 3 shows that switching to an index portfolio results in significant welfare improvement when investor $B$ 's absolute bias $\left|\theta_{k}^{B}\right|$ is small and investor $A$ 's prior variance $\nu$ is large. For example, when $\theta_{k}^{B}=-0.25$ and $\nu=0.1^{2}$, the welfare improves by $48 \%$. This result may seem counter-intuitive at first; how can an index portfolio provide better welfare than an optimal portfolio based on learning? The reason is two-fold. Firstly, although investor $A$ is perfectly rational, he has incomplete information about the true drifts, which means that his subjective and the objective probabilities do not coincide. Although his belief eventually converges to the truth, initially there can be large deviations from the truth especially when the prior variance is large. Secondly, when investor $B$ 's absolute bias is relatively small, it limits the potential gain from speculation for investor $A$. Note that in the limit as $\theta_{k}^{B} \rightarrow 0$, investor $A$ underperforms both $B$ and the index investor. In summary, the results in Table 3 illustrate that an index portfolio can potentially outperform an optimal portfolio strategy based on Bayesian learning in a general equilibrium framework.

We also use Monte-Carlo simulation to estimate the market selection time. Figure 6 shows that in this scenario investor $A$ eventually drive out investor $B$ in the long run and the market selection time increases with $A$ 's prior variance $\nu$. For example, when $\nu=0.01^{2}$, it takes 


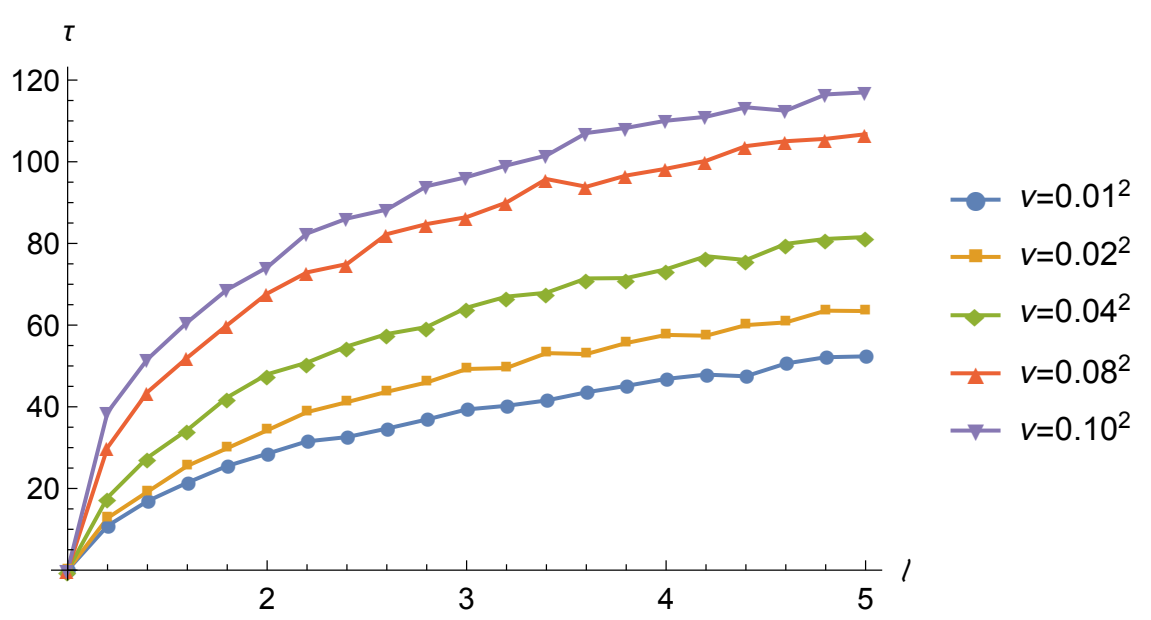

FIGURE 6. Speed of market selection $\tau=\mathbb{E}\left[\inf \left\{t: c_{A}^{*}(t) / c_{B}^{*}(t)=l\right\}\right]$ under Bayesian learning with constant drifts. Investor $B$ 's belief $\theta_{k}^{B}=-0.25, A$ 's initial prior $\mu_{k} \sim \mathcal{N}\left(\theta_{k}^{B}, \nu\right)$, subjective discount rate $\beta=0.02$, drift and volatility for the dividend processes are given by $\mu_{k}=0.02$ and $\sigma_{k}=0.02$ respectively, $k=1,2$.

$\tau \approx 52$ years for consumption ratio to reach $l=5$ (which is equivalent to investor $B$ losing $\frac{l}{1+l}-\frac{1}{2}=\frac{1}{3}$ of his consumption share to investor $A$ ). In comparison, when $\nu=0.1^{2}$, it takes $\tau \approx 117$ years. Therefore, when investor $A$ becomes more uncertain about his prior belief, the market selection can be quite slow.

4.2. Time-varying Drifts. We assume that in addition to the dividend process $D_{k}(t)$, the investors also observe a signal process $s_{k}(t)$. More specifically, following Dumas et al. (2009), the dividend processes evolve according to

$$
\begin{aligned}
\frac{d D_{k}(t)}{D_{k}(t)} & =\mu_{k}(t) d t+\sigma_{k} d Z_{k}(t) \\
d \mu_{k}(t) & =-\zeta_{k}\left(\mu_{k}(t)-\bar{\mu}_{k}\right) d t+\tilde{\sigma}_{k} d \tilde{Z}_{k}(t)
\end{aligned}
$$

and the public signal process follows

$$
d s_{k}(t)=\hat{\sigma}_{k}\left(\phi_{k} d \tilde{Z}_{k}(t)+\sqrt{1-\phi_{k}^{2}} d \hat{Z}_{k}(t)\right)
$$

for $k=1, \cdots, K$, where $Z_{k}(t), \tilde{Z}_{k}(t)$ and $\hat{Z}_{k}(t)$ are independent Wiener processes. The investors update their beliefs based on the filtration generation by $\mathcal{F}_{t}=\left\{D_{k}(t), s_{k}(t)\right\}$. However, they disagree about the parameter $\phi_{k}$, which measures the informativeness of the public signal. 
We denote their beliefs as $\phi_{k}^{i}, i=A, B$. Thus, investors' beliefs evolve according to

$$
d m_{k}^{i}(t)=-\zeta_{k}\left(m_{k}^{i}(t)-\bar{\mu}_{k}\right) d t+\frac{\nu_{k}^{i}}{\sigma_{k}^{2}}\left(\frac{d D_{k}(t)}{D_{k}(t)}-m_{k}^{i}(t) d t\right)+\phi_{k}^{i} \frac{\tilde{\sigma}_{k}}{\hat{\sigma}_{k}} d s_{k}(t)
$$

where $\nu_{k}^{i}$ is the steady state of agent $i$ 's posterior variance given by

$$
\nu_{k}^{i}=\sigma_{k}^{2}\left(\sqrt{\zeta_{k}^{2}+\left(1-\left(\phi_{k}^{i}\right)^{2}\right) \frac{\left(\tilde{\sigma}_{k}\right)^{2}}{\sigma_{k}^{2}}}-\zeta_{k}\right) .
$$

When $\phi_{k}^{i}=0$, investor $i$ pays no attention to the public signal and treats it as a pure noise. When $\phi_{k}^{i}=1$ investor $i$ behaves as if the underlying drifts are fully revealed by the public signal. We assume that under the objective probability measure $\mathcal{P}, \phi_{k}=0$. Therefore, investor $i$ 's deviation from the true drift is given by

$$
d \theta_{k}^{i}(t)=-\left(\zeta_{k}+\frac{\nu_{k}^{i}}{\sigma_{k}^{2}}\right) \theta_{k}^{i}(t) d t+\frac{\nu_{k}^{i}}{\sigma_{k}^{2}} d Z_{k}(t)+\phi_{k}^{i} \frac{\tilde{\sigma}_{k}}{\sigma_{k}} d \hat{Z}_{k}(t)-\frac{\tilde{\sigma}_{k}}{\sigma_{k}} d \tilde{Z}_{k}(t)
$$

for $k=1, \cdots, K$.

As in the case of the constant drifts, we use Monte-Carlo simulations (with the same time increment and horizon) to evaluate the welfare difference between investor $A$ and the index investor in (22) and report the results in Table 4. Because of the public signals, we need four risky assets to complete the market. We assume assets 1 and 2 are claims to the dividend processes with $N_{1}=1$ and $N_{2}=0$ while assets 3 and 4 are tradable, non-dividend paying assets in zero net supply with exogenous volatilities, which satisfy

$$
\frac{d S_{k}(t)}{S_{k}(t)}=\mu_{S, k}(t) d t+\boldsymbol{\sigma}_{S, k}^{\top} d \mathbf{Z}(t) \quad \text { for } k=3,4
$$

where $\mathbf{Z}(t)=\left(Z_{1}(t), Z_{2}(t), \hat{Z}_{1}(t), \hat{Z}_{2}(t)\right)^{\top}$. Furthermore, for simplicity, we assume $\phi_{k}^{B}=\phi$ and $\phi_{k}^{A}=\alpha \phi$ with $\alpha \in[-1,1), k=1,2$. Therefore, investor $A$ 's belief about the informativeness of the public signal is more accurate than that of $B$.

Table 4 leads to the following observations. Firstly, when investor $A$ correctly interprets the public signal (i.e. $\alpha=0$ ), switching to the index portfolio cannot improve the expected ex-post welfare for all the values of $\phi$ considered. Secondly, when investor $A$ misinterprets the signal, switching to the index portfolio can improve welfare when $\alpha \rightarrow-1$. The intuition is that when $\alpha \rightarrow-1$, not only are $A$ and $B$ 's interpretations equally inaccurate, their beliefs biases are also less correlated. From (49), they interpret dividend news in a similar way but public 


\begin{tabular}{ccccccc}
\hline$\alpha$ & -1.0 & -0.8 & -0.5 & 0.0 & 0.5 & 0.8 \\
\hline$\phi=0.1$ & 0.114 & 0.069 & 0.017 & -0.037 & -0.048 & -0.028 \\
$\phi=0.2$ & 0.262 & 0.162 & 0.025 & -0.117 & -0.150 & -0.100 \\
$\phi=0.5$ & 0.579 & 0.267 & -0.101 & -0.321 & -0.356 & -0.322 \\
$\phi=0.8$ & 0.785 & 0.092 & -0.303 & -0.405 & -0.423 & -0.418 \\
\hline
\end{tabular}

TABLE 4. The welfare improvement $\left(\mathcal{U}_{I}-\mathcal{U}_{A}\right) /\left|\mathcal{U}_{A}\right|$ by switching to an index portfolio under learning and time-varying drifts. Apart from $\alpha$ and $\phi$, other parameter values are given by $\beta=0.02, \sigma_{k}=0.02, \zeta_{k}=0.2, \bar{\mu}_{k}=0.015$ and $\tilde{\sigma}_{k}=0.03$ for $k=1,2$.

signal in the opposite way. In this case, the speculation makes neither $A$ and $B$ better off since it only increases their consumption volatility. Secondly, the welfare improvement becomes increasingly significant when $\alpha \rightarrow-1$ and $\phi \rightarrow 1$. For example, when $\alpha=-1$ and $\phi=0.8$, switching to the index portfolio improves the expected ex-post welfare by a massive $78.5 \%$. However, when $A$ interprets the signal more accurately, for example when $\alpha=-0.8$ and $\phi=0.8$, the welfare improvement drops to just $9.2 \%$. In summary, we show that our baseline results are robust even in a more complex learning environment as we identify scenarios under which an index portfolio leads to significant welfare improvement over the active investing.

Figure 7 plots the market-selection time for different values of $\phi$ with $\alpha=-0.8$ in plot (a) and different values of $\alpha$ with $\phi=0.5$ in plot (b). Firstly, plot (a) shows that the market selection can be very fast when $\phi \rightarrow 1$. For example, when $\phi=0.8$, it takes only $\tau \approx 5$ years for investor $B$ to lose $1 / 3$ of consumption share to $A$ (i.e. $l=5$ ). In comparison, it takes 155 years when $\phi=0.1$. Secondly, plot (b) shows that the market selection time is less sensitive to $\alpha$. For example, the market selection time for $l=5$ increases slightly from 8.6 to 10.3 years when $\alpha$ increases from 0 to 0.5 . Furthermore, plot (b) also shows that only the absolute value of $\alpha$ matters and not its sign. Intuitively, investor $A$ becomes perfectly rational when $|\alpha| \rightarrow 0$ and equally irrational as $B$ when $|\alpha| \rightarrow 1$. In contrast, the sign of $\alpha$ matters significantly for the expected ex-post welfare as we have shown in Table 4.

\section{A More Volatile Index Portfolio}

We relax the assumption that the index coincides with the market portfolio whose dividend process is exactly the aggregate endowment. We assume that the aggregate endowment process is given by

$$
D(t)=D_{1}(t)+D_{2}(t)
$$




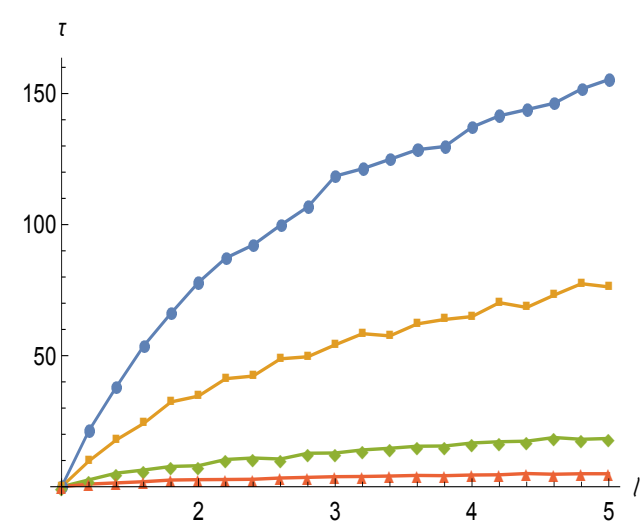

(a) $\phi \in[0.1,0.8]$
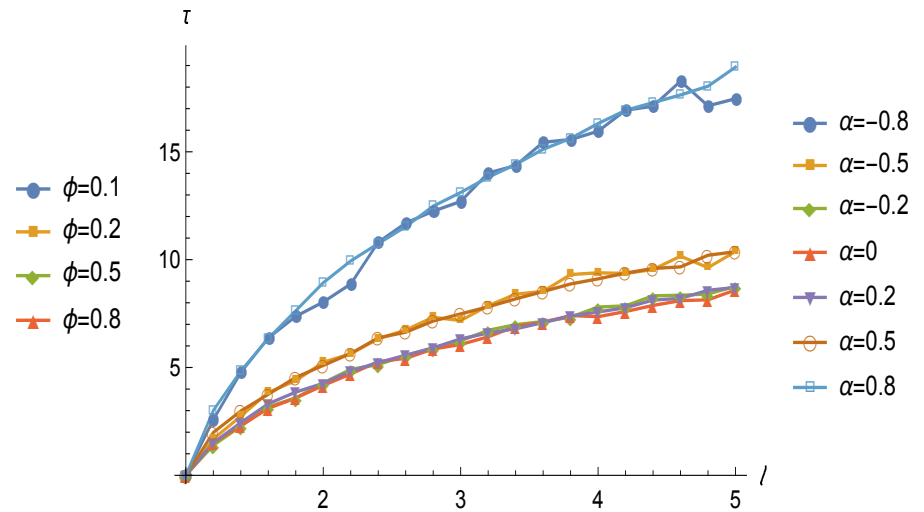

(b) $\alpha \in[-0.8,0.8]$

FIGURE 7. Market-selection time $\tau=\mathbb{E}\left[\inf \left\{t: c_{A}^{*}(t) / c_{B}^{*}(t)=l\right\}\right]$ under sentiment learning with time-varying drifts, $\alpha=-0.8$ and $\phi \in[0.1,0.8]$ in plot (a), $\phi=0.5$ and $\alpha \in[-0.8,0.8]$ in plot (b). Other parameter values are given by $\beta=0.02, \sigma_{k}=0.02, \zeta_{k}=0.2, \bar{\mu}_{k}=0.015$ and $\tilde{\sigma}_{k}=0.03$ for $k=1,2$.

where $D_{1}(t)$ and $D_{2}(t)$ are geometric Brownian motions as described in (6) with drifts $\mu_{k}$ and volatilities $\sigma_{k}$ with $k=1,2$. Moreover, index investor $I$ holds stock 2 and consumes its dividend, thus $c_{I}(t)=D_{2}(t)$. Furthermore, we assume $\sigma_{1} \leq \sigma_{2}$, thus index investor's consumption growth is more volatile than that of the aggregate endowment.

Investors $A$ and $B$ have logarithmic preferences and constant beliefs about the drifts as in our baseline model. Since the optimal consumptions of the active portfolios are given by (15) and market clearing condition requires that $c_{A}^{*}(t)+c_{B}^{*}(t)=D_{1}(t)$. It is straightforward to show that the state price density is given by

$$
\xi(t)=M(t) e^{-\beta t} \frac{1}{D_{1}(t)}
$$

where $M(t)$ characterizes the consensus probability belief as in (19). Using the state price density in (53), we can compute the initial wealth of the index investor 17 ,

$$
W_{I}(0)=\mathbb{E}_{0}\left[\int_{0}^{\infty} \frac{\xi(t)}{\xi(0)} D_{2}(t) d t\right]=\frac{x_{A}}{\beta+\sigma_{1}^{2}+\left(\mu_{2}^{A}-\mu_{1}^{A}\right)}+\frac{x_{B}}{\beta+\sigma_{1}^{2}+\left(\mu_{2}^{B}-\mu_{1}^{B}\right)} .
$$

For simplicity, we assume for each investor, $\mu_{1}^{i}=\mu_{2}^{i}$ for $i=A, B$, thus $W_{I}(0)$ no longer depends on the subjective beliefs. Next, we compute the welfare of the index investor in closedform according to Definition 2.3 ,

$$
\mathcal{U}_{I}=\frac{1}{\beta} \ln \left(\beta+\sigma_{1}^{2}\right)+\frac{1}{\beta^{2}}\left(\mu_{2}-\sigma_{2}^{2} / 2\right)
$$

\footnotetext{
${ }^{17}$ We assume that the initial values are given by $D_{k}(0)=1$ for $k=1,2$.
} 
and also the welfare of investor $A$,

$$
\mathcal{U}_{A}=\frac{1}{\beta} \ln (\beta)+\frac{1}{\beta^{2}}\left(\mu_{1}-\sigma_{1}^{2} / 2\right)-\mathbb{E}_{0}\left[\int_{0}^{\infty} e^{-\beta t} \ln \left(x_{A}+x_{B} \eta(t)^{-1}\right) d t\right] .
$$

Equations (55) and (56) show that one part of the difference in the welfare is due to speculation, another part is due to under-diversification - the consumption growth of the index portfolio is more volatile than the aggregation consumption growth. We compute $\mathcal{U}_{A}$ in (56) using numerical integration assuming that $x_{A}=x_{B}=\frac{1}{2}$. Furthermore, we assume $\theta_{k}^{A}=-\alpha \theta_{k}^{B}, k=1,2$, such that the bias correlation $\rho=-1$ and the absolute bias ratio $\vartheta=\alpha$.

\begin{tabular}{ccccccc}
\hline$\alpha$ & 0.01 & 0.2 & 0.4 & 0.6 & 0.8 & 1.0 \\
\hline$\sigma_{2}=0.02$ & -0.215 & -0.195 & -0.155 & -0.073 & 0.081 & 0.305 \\
$\sigma_{2}=0.05$ & -0.237 & -0.217 & -0.176 & -0.093 & 0.064 & 0.292 \\
$\sigma_{2}=0.10$ & -0.315 & -0.294 & -0.251 & -0.162 & 0.005 & 0.247 \\
$\sigma_{2}=0.15$ & -0.446 & -0.423 & -0.375 & -0.278 & -0.094 & 0.172 \\
\hline
\end{tabular}

TABLE 5. The welfare improvement $\left(\mathcal{U}_{I}-\mathcal{U}_{A}\right) /\left|\mathcal{U}_{A}\right|$ under logarithmic utility and a more volatile index portfolio. Investors' beliefs are given by $\theta_{k}^{B}=-0.25$ and $\theta_{k}^{A}=-\alpha \theta_{k}^{B}, k=1,2$. Other parameter values are given by $\beta=0.02$, $\sigma_{1}=0.02$ and $\mu_{k}=0.02$ for $k=1,2$.

Table 5 shows that a more volatile index portfolio reduces the potential welfare improvement by switching to the index portfolio. However, when the investors are equally biased (i.e. $\alpha=$ $1)$, the welfare improvements $\left(\mathcal{U}_{I}-\mathcal{U}_{A}\right) /\left|\mathcal{U}_{A}\right|=24.7 \%$ when $\sigma_{2}=0.10$ and $17.2 \%$ when $\sigma_{2}=0.15$. Therefore, although the welfare of the index portfolio deteriorates due to underdiversification, it can still provide a significant welfare improvement when the active investors have negatively correlated biases with the same magnitude.

Next, instead of constant beliefs, we assume that investor $A$ updates his belief and learns about the drifts over time (as in Section 4.1) with his priors given by $\mu_{k}^{A}(0) \sim \mathcal{N}\left(\mu_{k}^{B}, \nu\right)$ for $k=1,2$. Note that, assume $\mu_{1}^{B}=\mu_{2}^{B}$, the initial wealth of the index portfolio in this case is given by

$$
W_{I}(0)=\frac{x_{B}}{\beta+\sigma_{1}^{2}}+x_{A} \mathbb{E}_{0}^{A}\left[\int_{0}^{\infty} e^{-\beta t} \frac{D_{2}(t)}{D_{1}(t)} d t\right]
$$

and his welfare is given by

$$
\mathcal{U}_{I}=\frac{1}{\beta^{2}}\left(\mu_{2}-\sigma_{2}^{2} / 2\right)-\frac{1}{\beta} \ln \left[W_{I}(0)\right]
$$


As in Section 4.1, we compute $\mathcal{U}_{A}$ in (56) and $W_{I}(0)$ using Monte-Carlo simulation with $\Delta t=$ $0.01, T=350$ and 10,000 simulated paths.

\begin{tabular}{cccccc}
\multicolumn{6}{c}{ Panel A: $\left(\mathcal{U}_{I}-\mathcal{U}_{A}\right) /\left|\mathcal{U}_{A}\right|$} \\
\hline$\nu$ & $0.01^{2}$ & $0.02^{2}$ & $0.04^{2}$ & $0.08^{2}$ & $0.10^{2}$ \\
\hline$\sigma_{2}=0.02$ & -0.029 & 0.115 & 0.277 & 0.427 & 0.471 \\
$\sigma_{2}=0.05$ & -0.059 & 0.005 & 0.133 & 0.298 & 0.348 \\
$\sigma_{2}=0.10$ & -0.048 & -0.067 & -0.084 & 0.007 & 0.014 \\
$\sigma_{2}=0.15$ & -0.107 & -0.113 & -0.383 & -0.793 & -1.256 \\
\hline \multicolumn{6}{c}{ Panel B: $\ln \left[W_{I}(0)\right]$} \\
\hline$\nu$ & $0.01^{2}$ & $0.02^{2}$ & $0.04^{2}$ & $0.08^{2}$ & $0.10^{2}$ \\
\hline$\sigma_{2}=0.02$ & 3.944 & 3.947 & 3.953 & 3.954 & 3.950 \\
$\sigma_{2}=0.05$ & 3.855 & 3.960 & 4.039 & 4.068 & 4.079 \\
$\sigma_{2}=0.10$ & 3.660 & 3.894 & 4.374 & 4.775 & 5.050 \\
$\sigma_{2}=0.15$ & 3.545 & 3.746 & 4.974 & 7.368 & 9.810 \\
\hline
\end{tabular}

TABLE 6. The welfare improvement $\left(\mathcal{U}_{I}-\mathcal{U}_{A}\right) /\left|\mathcal{U}_{A}\right|$ under logarithmic utility and a more volatile index portfolio. Investor $B$ 's belief is given by $\theta_{k}^{B}=-0.25$ and $\theta_{k}^{A}=-\alpha \theta_{k}^{B}$. Investor $A$ learns about the drifts with prior $\mu_{k}^{A}(0) \sim \mathcal{N}\left(\mu_{k}^{B}, \nu\right)$ for $k=1,2$. Other parameter values are given by $\beta=0.02, \sigma_{1}=0.02$ and $\mu_{k}=0.02$.

When compared to Table 3, Table 6 panel A shows that a larger prior variance $\nu$ no longer monotonically increases the welfare improvement by switching to an index portfolio. When the consumption growth the index portfolio becomes very volatile, for example $\sigma_{2}=0.15$, an increasing in the prior variance actually further deteriorates its welfare relative to that of investor $A$. The reason is as follows. As $\nu$ increases, although the speculation makes the consumption growth of the active investors more volatile, it also makes the index portfolio initially more expensive (i.e. $W_{I}(0)$ increases). Note that when the index portfolio coincides with the market portfolio, its value is fixed at $W_{I}(0)=1 / \beta$ (i.e. unaffected by agents' subjective beliefs), which is no longer true here. Table 6 panel B shows that $W_{I}(0)$ is monotonically increasing in both $\nu$ and $\sigma_{2}$. Therefore, in the case of learning there is a trade off between the initial cost of the index portfolio and the welfare improvement it can potentially provide over the active portfolios.

\section{CONCLUSION}

We show that, in terms of the welfare measured by the expected realized (ex-post) utility, an index portfolio can dominate active investing. Our baseline model has logarithmic preference and constant beliefs, we also consider extensions to CRRA preference, learning under incomplete information and an alternative specification of the index portfolio. 
The key intuition is that the deviations from the truth (either due to biases or incomplete information) are detrimental to the expected ex-post welfare and their negative impact is amplified by the disagreement between active investors, which makes their consumption shares more volatile. Therefore, although an active investor may have a higher consumption growth than an index portfolio in the long-run, it does not necessarily achieve a better expected ex-post welfare due to an imperfect balance between speculation and risk-sharing. Moreover, we show that a higher risk aversion works in favor of the index investor since it increases the concavity of the utility function, although it also reduces the consumption share volatility, the first effect tends to dominate. The magnitude of the welfare improvement also increases with higher risk aversion. Furthermore, a more volatile index portfolio reduces the magnitude of the welfare improvement.

Admittedly, our model is stylized and can be extended in several ways. In a recent theoretical paper, Chabakauri and Rytchkov (2014) constrain one group of agents to only investing in an index portfolio and the risk-free security and study the implication on asset prices in a general equilibrium model. In our model, index portfolio cannot be combined with lending or borrowing, thus do not affect equilibrium prices. It would be interesting to look at whether investing in the risk-free security helps to further improve the expected ex-post welfare of index investors. More broadly, one can examine the welfare improving potential for any fixed portfolio-consumption strategies. However, in those cases the proportion of investors adopting the index or fixed strategies will affect market equilibrium, the feedback effect will complicate the welfare analysis. The switch to an index portfolio may also be triggered by the cost of active investing or social interaction. Moreover, institutional and retail investors can be modelled differently, for example, the former may care about their performance relative to a certain benchmark (Basak and Pavlova 2013). Furthermore, one can also consider the impact of portfolio delegation and interaction between principals (investors) and agents (fund managers) on market equilibrium and investors' welfare (Vayanos and Woolley 2013, Buffa, Vayanos and Woolley 2014). Lastly, our model and many other differences-in-opinion models converge to a singleagent economy in the long run. Therefore, in order to have a stationary market equilibrium, one could consider an overlapping generations model (Ehling, Graniero and Heyerdahl-Larson 2014), recursive preferences (Borovicka 2015) or market frictions (Chabakauri and Han 2016). We leave these extensions for future research. 


\section{APPENDiX A. PRoOfS}

A.1. Proof of Proposition 2.5, We first prove Lemma 2.4. Under logarithmic utility, we obtain

$$
\ln \left(\frac{c_{I}^{*}(t)}{W_{I}(0)}\right)-\ln \left(\frac{c_{A}^{*}(t)}{W_{A}(0)}\right)=\ln \left(\frac{D(t)}{W_{M}(0)}\right)-\ln \left(\frac{c_{A}^{*}(t)}{W_{A}(0)}\right) .
$$

However, the aggregate dividend $D(t)$ is the optimal consumption for the representative agent with the consensus belief $M(t)$ defined in (19). Therefore,

$$
\ln \left(\frac{c_{I}^{*}(t)}{W_{I}(0)}\right)-\ln \left(\frac{c_{A}^{*}(t)}{W_{A}(0)}\right)=-\ln \left(\frac{M_{A}(t)}{M(t)}\right)=\ln \left(x_{A}+x_{B} \eta(t)^{-1}\right),
$$

where $x_{A}=W_{A}(0) /\left(W_{A}(0)+W_{B}(0)\right)$ and $x_{B}=1-x_{A}$, and $\eta(t)$ is given by Equation (16).

By Jensen's inequality,

$$
\left.\mathbb{E}\left[\ln \left(x_{A}+x_{B} \eta(t)^{-1}\right)\right] \leq \ln \left(x_{A}+x_{B} \mathbb{E}\left[\eta(t)^{-1}\right]\right)\right],
$$

where

or

$$
\mathbb{E}\left[\eta(t)^{-1}\right]=\exp \left\{\frac{1}{2}\left(\left\|\boldsymbol{\theta}_{A}\right\|^{2}-\left\|\boldsymbol{\theta}_{B}\right\|^{2}+\|\Delta \boldsymbol{\theta}\|^{2}\right) t\right\}, \quad \Delta \boldsymbol{\theta}=\boldsymbol{\theta}_{A}-\boldsymbol{\theta}_{B},
$$

$$
\mathbb{E}\left[\eta(t)^{-1}\right]=\exp \left\{\frac{1}{2}\left\|\boldsymbol{\theta}_{A}\right\|^{2}\left(1-\rho \frac{\left\|\boldsymbol{\theta}_{B}\right\|}{\left\|\boldsymbol{\theta}_{A}\right\|}\right) t\right\} .
$$

Therefore, since $x_{A}>0, x_{B}>0$ and $x_{A}+x_{B}=1$, a sufficient condition for $\mathcal{U}_{I}<\mathcal{U}_{A}$ is given by $\mathbb{E}\left[\eta(t)^{-1}\right] \leq 1$, which is equivalent to

which simplifies to condition (S1).

$$
1<\rho \frac{\left\|\boldsymbol{\theta}_{B}\right\|}{\left\|\boldsymbol{\theta}_{A}\right\|}
$$

In the case of $\left\|\boldsymbol{\theta}_{A}\right\|=\left\|\boldsymbol{\theta}_{B}\right\|$, from (16), $\eta(t)$ and $\eta(t)^{-1}$ have the same probability distribution. Therefore, we have from (58) and the assumption $W_{A}(0)=W_{B}(0)$ that

$$
\frac{1}{2} \frac{M_{A}(t)}{M(t)}+\frac{1}{2} \frac{M_{B}(t)}{M(t)}=1
$$

By symmetry, we obtain

$$
\mathbb{E}_{0}\left[\frac{M_{A}(t)}{M(t)}\right]=\mathbb{E}_{0}\left[\frac{M_{B}(t)}{M(t)}\right]=1
$$

By Jensen's inequality,

$$
\mathbb{E}_{0}\left[\ln \left(\frac{c_{A}^{*}(t)}{W_{A}(0)}\right)-\ln \left(\frac{c_{I}^{*}(t)}{W_{I}(0)}\right)\right]<\ln \left(\mathbb{E}_{0}\left[\frac{M_{A}(t)}{M(t)}\right]\right)=0
$$

and the same holds for investor $B$. Hence, $\mathcal{U}_{A}<\mathcal{U}_{I}$.

A.2. Proof of Lemma 2.6. Since the consumption ratio between investors $A$ and $B$ is given by

$$
\frac{c_{A}^{*}(t)}{c_{B}^{*}(t)}=\frac{x_{A} M_{A}(t)}{x_{B} M_{B}(t)}=\frac{x_{A}}{x_{B}} \eta(t),
$$

an equivalent problem to computing (25) is to compute

$$
\tau=\mathbb{E}_{0}\left[\inf \left\{t: a t+Z_{t}=l^{*}\right\}\right],
$$

where $Z(t)$ is a Wierner process and

$$
a=\frac{1}{2} \frac{\left\|\boldsymbol{\theta}_{B}\right\|^{2}-\left\|\boldsymbol{\theta}_{A}\right\|^{2}}{\left\|\boldsymbol{\theta}_{A}-\boldsymbol{\theta}_{B}\right\|} \text { and } l^{*}=\frac{\ln (l)-\ln \left(x_{A} / x_{B}\right)}{\left\|\boldsymbol{\theta}_{A}-\boldsymbol{\theta}_{B}\right\|} .
$$


This is a well studied problem, see Karatzas and Shreve (1991) Chapter 3.5, and the explicit density function of $\tau_{l}$ is given by

$$
\mathcal{P}[l \in d t]=\frac{\left|l^{*}\right|}{\sqrt{2 \pi t^{3}}} \exp \left\{-\frac{\left(l^{*}-a t\right)^{2}}{2 t}\right\} d t, t>0 .
$$

To find the expected first hitting time, we compute the integral

$$
\int_{0}^{\infty} \frac{\left|l^{*}\right|}{\sqrt{2 \pi t}} \exp \left\{-\frac{\left(l^{*}-a t\right)^{2}}{2 t}\right\} d t
$$

The explicit solution of (60) depends on the sign of $a$ and $l^{*}$. Since $a$ and $l^{*}$ are both positive, the integral in (60) has the closed-form solution given by

$$
\tau=\frac{l^{*}}{a}
$$

Substituting in the values for $a$ and $l^{*}$ completes the proof.

A.3. Proof of Lemma 3.1. We first prove that investors $A$ and $B$ have the same initial consumption. From the market clearing condition that $c_{A}^{*}(t)+c_{B}^{*}(t)+c_{I}^{*}(t)=D_{1}(t)$, the state price density process is given by

$$
\xi(t)=e^{-\beta t}\left(x_{A} M_{A}(t)^{\frac{1}{\gamma}}+x_{B} M_{B}(t)^{\frac{1}{\gamma}}\right)^{\gamma}\left(\frac{D_{1}(t)}{D_{1}(0)}\right)^{-\gamma},
$$

where $x_{A}$ and $x_{B}$ are defined in (37). Note that the initial wealth of investor $A$ is given by

$$
W_{A}(0)=\mathbb{E}_{0}\left[\int_{0}^{\infty} \xi(t) c_{A}^{*}(t) d t\right],
$$

which, after some algebra, from (33), can be expressed under $A$ 's subjective probability measure $\mathcal{P}_{A}$ as

$$
W_{A}(0)=\mathbb{E}_{0}^{A}\left[\int_{0}^{\infty} e^{-\beta t}\left(x_{A}+x_{B} \eta(t)^{-\frac{1}{\gamma}}\right)^{\gamma-1}\left(\frac{D_{1}(t)}{D_{1}(0)}\right)^{-\gamma} d t\right] .
$$

Similarly, we can express $B$ 's initial wealth under $\mathcal{P}_{B}$ as

$$
W_{B}(0)=\mathbb{E}_{0}^{B}\left[\int_{0}^{\infty} e^{-\beta t}\left(x_{B}+x_{A} \eta(t)^{\frac{1}{\gamma}}\right)^{\gamma-1}\left(\frac{D_{1}(t)}{D_{1}(0)}\right)^{-\gamma} d t\right] .
$$

Note that under $\mathcal{P}_{A}, \mathbf{Z}_{A}(t) \equiv \mathbf{Z}(t)-\boldsymbol{\theta}_{A} t$ is a Brownian motion, and

$$
\eta(t)=\exp \left\{\frac{1}{2}\|\Delta \boldsymbol{\theta}\|^{2} t+\Delta \boldsymbol{\theta}^{\top} \mathbf{Z}_{A}(t)\right\} .
$$

Similarly, $\eta(t)^{-1}$ under the probability measure $\mathcal{P}_{B}$ has the same probability distribution as $\eta(t)$ under $\mathcal{P}_{A}$, that is

$$
\eta(t)^{-1}=\exp \left\{\frac{1}{2}\|\Delta \boldsymbol{\theta}\|^{2} t+\Delta \boldsymbol{\theta}^{\top} \mathbf{Z}_{B}(t)\right\} .
$$

Therefore, since $A$ and $B$ agree on the aggregate consumption process, $\theta_{1}^{A}=\theta_{1}^{B}=\theta_{1}$, we have $W_{A}(0)=W_{B}(0)$ if and only if $x_{A}=x_{B}=\frac{1}{2}$, which implies that $\lambda_{A}(0)=\lambda_{B}(0)$.

Next, given that $x_{A}=x_{B}=\frac{1}{2}$ when $\theta_{1}^{A}=\theta_{1}^{B}=\theta_{1}$ and $W_{A}(0)=W_{B}(0)$, the expected instantaneous utility of $A$, in (38), is given by

$$
\frac{1}{1-\gamma} \mathbb{E}_{0}\left[{\frac{c_{A}^{*}(t)}{W_{A}(0)}}^{1-\gamma}\right]=\frac{1}{1-\gamma} \mathbb{E}_{0}\left[\left(\frac{1}{2}\left(1+\eta(t)^{-\frac{1}{\gamma}}\right)\right)^{\gamma-1}{\frac{c_{I}^{*}(t)}{W_{I}(0)}}^{1-\gamma}\right] .
$$

Let $X(t) \equiv(\Delta \boldsymbol{\theta})^{\top} \mathbf{Z}(t)$ and $Y(t) \equiv \sigma_{1} Z_{1}(t)$. Since $X(t)$ and $Y(t)$ are bivariate normal with $\mathbb{E}[X(t)]=$ $\mathbb{E}[Y(t)]=0, \operatorname{Var}[X(t)]=\|\Delta \boldsymbol{\theta}\|^{2} t, \operatorname{Var}[Y(t)]=\sigma_{1}^{2} t$ and $\operatorname{Correl}[X(t), Y(t)]=\frac{\Delta \theta_{1}}{\|\Delta \boldsymbol{\theta}\|}$. Therefore, 
the conditional distribution of $Y(t)$ is given by

$$
Y(t) \mid(X(t)=x) \sim \mathcal{N}\left(\frac{\sigma_{1} \Delta \theta_{1}}{\|\Delta \boldsymbol{\theta}\|^{2}} x,\left(1-\frac{\Delta \theta_{1}^{2}}{\|\Delta \boldsymbol{\theta}\|^{2}}\right) \sigma_{1}^{2} t\right)
$$

When $\Delta \theta_{1}=0$, from Equation (62), $Y(t)$ is independent of $X(t)$, thus $D_{1}(t)$ is independent of $\eta(t)$. This completes the proof.

A.4. Proof of Proposition 3.2, Let $f(x)=x^{\gamma-1}$. Since $f^{\prime \prime}(x)=(\gamma-1)(\gamma-2) x^{\gamma-3}$, we have

$$
f^{\prime \prime}(x) \begin{cases}<0, & 1<\gamma<2 \\ =0, & \gamma=2 \\ >0, & 0<\gamma<1 \text { or } \gamma>2\end{cases}
$$

Therefore, by Jensen's inequality,

$$
\mathbb{E}\left[\left(\frac{1}{2}\left(1+\eta(t)^{-\frac{1}{\gamma}}\right)\right)^{\gamma-1}\right]-\left(\mathbb{E}\left[\frac{1}{2}\left(1+\eta(t)^{-\frac{1}{\gamma}}\right)\right]\right)^{\gamma-1} \begin{cases}<0, & 1<\gamma<2 \\ =0, & \gamma=2 \\ >0, & 0<\gamma<1 \text { or } \gamma>2\end{cases}
$$

where

$$
\left(\mathbb{E}\left[\frac{1}{2}\left(1+\eta(t)^{-\frac{1}{\gamma}}\right)\right]\right)^{\gamma-1}=\left(\frac{1}{2}\left(1+\exp \left\{\frac{1}{2 \gamma}\left(\left\|\boldsymbol{\theta}_{A}\right\|^{2}-\left\|\boldsymbol{\theta}_{B}\right\|^{2}+\frac{1}{\gamma}\|\Delta \boldsymbol{\theta}\|^{2}\right)\right\}\right)\right)^{\gamma-1} .
$$

Then $\left\|\boldsymbol{\theta}_{B}\right\|^{2}-\left\|\boldsymbol{\theta}_{A}\right\|^{2} \geq \frac{1}{\gamma}\|\Delta \boldsymbol{\theta}\|^{2}$ is sufficient for $\mathbb{E}\left[\frac{c_{A}^{*}(t)^{1-\gamma}}{1-\gamma}\right]>\mathbb{E}\left[\frac{c_{I}^{*}(t)^{1-\gamma}}{1-\gamma}\right]$ when $0<\gamma<2$. Also $\left\|\boldsymbol{\theta}_{B}\right\|^{2}-\left\|\boldsymbol{\theta}_{A}\right\|^{2}>\frac{1}{\gamma}\|\Delta \boldsymbol{\theta}\|^{2}$ is a necessary and sufficient condition when $\gamma=2$ and a necessary condition when $\gamma>2$. The condition $\left\|\boldsymbol{\theta}_{B}\right\|^{2}-\left\|\boldsymbol{\theta}_{A}\right\|^{2} \geq \frac{1}{\gamma}\|\Delta \boldsymbol{\theta}\|^{2}$ is equivalent to the following quadratic inequality,

$$
\frac{1}{2}(1+\gamma) \vartheta^{2}-\rho \vartheta-\frac{1}{2}(\gamma-1) \leq 0
$$

where $\vartheta=\left\|\boldsymbol{\theta}_{A}\right\| /\left\|\boldsymbol{\theta}_{B}\right\|$, (63) has two roots $L$ and $R$ defined in Equation (41) if and only if $\gamma \geq \sqrt{1-\rho^{2}}$. When $\gamma=\sqrt{1-\rho^{2}}, L=R=\rho /(1+\gamma)$. Since the quadratic in (41) is convex, the inequality holds when $L \leq \vartheta \leq R$. 


\section{REFERENCES}

Admati, A. (1985), 'A noisy rational expectations equilibrium for multi-asset security markets', Econometrica 53, 629-657.

Anderson, E., Ghysels, E. and Juergens, J. (2005), 'Do heterogeneous beliefs matter for asset pricing?', Review of Financial Studies 18(3), 875-924.

Atmaz, A. and Basak, S. (2015), Belief dispersion in the stock market. SSRN Working Paper.

Banerjee, S. (2011), 'Learning from prices and the dipsersion in beliefs', Review of Financial Studies 24, 30253068 .

Banerjee, S. and Kremer, I. (2010), 'Disagreement and learning: Dynamic patterns of trade', Journal of Finance 65, 1269-1302.

Barberis, N. and Xiong, W. (2012), 'Realization utility', Journal of Financial Economics 104, 251-271.

Basak, S. (2000), 'A model of dynamic equilibrium asset pricing with heterogeneous beliefs and extraneous beliefs', Journal of Economic Dynamics and Control 24, 63-95.

Basak, S. (2005), 'Asset pricing with heterogeneous beliefs', Journal of Banking and Finance 29, 2849-2881.

Basak, S. and Pavlova, A. (2013), 'Asset prices and institutional investors', American Economic Review 103, 17281758.

Berraday, T. (2009), 'Bounded rationality and asset pricing with intermediate consumption', Review of Finance 13, 693-725.

Bhamra, H. and Uppal, R. (2014), 'Asset prices with heterogeneity in preferences and beliefs', Review of Financial Studies 27, 519-580.

Biais, B., Bossaerts, P. and Spatt, C. (2010), 'Equilibrium asset pricing and portfolio choice under asymmetric information', Review of Financial Studies 23, 1503-1543.

Blume, L. and Easley, D. (2006), 'If you are so smart, why aren't you rich? belief selection in complete and incomplete markets', Econometrica 74, 929-966.

Blume, L. and Easley, D. (2009a), Handbook of Financial Markets: Dynamics and Evoluation, Elsevier, chapter Market Selection and Asset Pricing, 403-424.

Blume, L. and Easley, D. (2009b), 'The market organism: Long-runsurvival in markets with heterogeneous traders', Journal of Economic Dynamics and Control 33, 1023-1035.

Borovicka, J. (2015), Survival and long-run dynamics with heterogeneous beliefs under recursive preferences. Working Paper, New York University.

Branger, N., Schlag, C. and Wu, L. (2015), 'Nobody is perfect: Asset pricing and long-run survival when heterogeneous investors exhibit different kinds of filtering errors', Journal of Economic Dynamics and Control 61, 303-333.

Brunnermeier, M. K. and Parker, J. A. (2005), 'Optimal expectations', American Economic Review 94, 159-165. 
Brunnermeier, M., Simsek, A. and Xiong, W. (2014), 'A welfare criterion for models with distorted beliefs', Quarterly Journal of Economics pp. 1753-1797.

Buffa, A., Vayanos., D. and Woolley, P. (2014), Asset management contracts and equilibrium prices. Working paper, LSE.

Buraschi, A. and Jiltsov, A. (2006), 'Model uncertianty and option markets with heterogeneous beliefs', Journal of Finance 61, 2814-2897.

Busse, J., Goyal, A. and Wahal, S. (2010), 'Performance and persistence in institutional investment management', Journal of Finance 65, 765-790.

Chabakauri, G. and Han, B. (2016), Captial requirements and asset prices. Working Paper, London School of Economics.

Chabakauri, G. and Rytchkov, O. (2014), Asset pricing with index investing. SSRN Working Paper.

Cremers, K. and Petajisto, A. (2009), 'How active is your fund manager? A new measure that predicts performance.', Review of Financial Studies 22, 3330-3365.

Cremers, M., Ferreira, M., Matos, P. and Starks, L. (2011), The mutual fund industry worldwide: Explicit and closet indexing, fees, and performance. Working Paper, Yale School of Management.

Cvitanić, J., Jouini., E., Malamud., S. and Napp, C. (2012), 'Financial markets equilibrium with heterogeneous agents', Review of Finance 16, 285-321.

Cvitanić, J. and Malamud, S. (2011), 'Price impact and portfolio impact', Journal of Financial Economics 100, 201-225.

David, A. (2008), 'Heterogeneous beliefs, speculation, and the equity premium', Journal of Finance 63, 41-83.

Detemple, J. and Murthy, S. (1994), 'Intertemporal asset pricing with heterogeneous beliefs', Journal of Economic Theory 62, 294-320.

Dumas, B., Kurshev, A. and Uppal, R. (2009), 'Equilibrium portfolio strategies in the presence of sentiment risk and excess volatility', Journal of Finance 64, 195-229.

Dyck, A., Lins, K. and Pomorski, L. (2013), 'Does active management pay? New international evidence', Reivew of Asset Pricing Studies 3, 200-228.

Ehling, P., Graniero., A. and Heyerdahl-Larsen, C. (2014), Asset prices and portfolio choices with learning from experience. Working paper, LBS.

Fama, E. and French, K. (1993), 'Common risk facors in the returns on stocks and bonds', Journal of Financial Economics 33, 3-56.

Fedyk, Y., Heyerdahl-Larsen, C. and Walden, J. (2013), 'Market selection and welfare in a multi-asset economy', Review of Finance 17, 1179-1237.

French, K. (2008), 'Presidential address: The cost of active investing', Journal of Finance 63, 1537-1573.

Grossman, S. and Stiglitz, J. (1980), 'On the impossibility of informationally efficient markets', American Economic Review 70, 393-408.

Gruber, M. (1996), 'The growth in actively managed mutual funds', Journal of Finance 51, 783-810. 
Hammond, P. (1981), 'Ex-ante and ex-post welfare optimality under uncertainty’, Economica 48, $235-250$.

Hansen, S. (2015), 'Cross-sectional asset pricing with heterogeneous preferences and beliefs', Journal of Economic Dynamics and Control 58, 125-151.

Harris, R. (1978), 'Ex-post efficiency and resource allocation under uncertainty', Review of Economics and Statistics 45, 427-436.

Hausman, D. and McPherson, M. (1994), 'Preference, belief, and welfare', American Economic Review 84, 396400.

Jensen, M. (1968), 'The performance of mutual funds in the period 1945-1964', Journal of Finance 23, $389-416$.

Jouini, E. and Napp, C. (2006), 'Heterogeneous beliefs and asset pricing in discrete time: An analysis of pessimism and doubt', Journal of Economic Dynamics and Control 30, 1233-1260.

Jouini, E. and Napp, C. (2007), 'Consensus consumer and intertemporal asset pricing with heterogeneous beliefs', Review of Economic Studies 74, 1149-1174.

Jouini, E. and Napp, C. (2011), 'Unbiased disagreement in financial markets, waves of pessimism and the riskreturn trade-off', Review of Finance 15, 575-601.

Karatzas, I. and Shreve, S. (1991), Brownian Motion and Stochastic Calculus (2nd Ed), Springer, New York.

Kogan, L., Ross, S., Wang, J. and Westerfield, M. (2006), 'The price impact and survival of irrational traders', Journal of Finance 61, 195-229.

Kogan, L., Ross, S., Wang, J. and Westerfield, M. (2012), Market selection. NBER Working Paper No. 15189.

Li, T. (2007), 'Heterogeneous beliefs, asset prices, and volatility in a pure exchange economy', Journal of Economic Dynamics and Control 31, 1697-1727.

Muraviev, R. (2013), 'Market selection with learning and catching up with the Joneses', Finance and Stochastics 17, 273-304.

Petajisto, A. (2013), ‘Active share and mutual fund performance’, Financial Analysts Journal 69, 73-93.

Sandroni, A. (2000), 'Do markets favor agents able to make accurate predictions?', Econometrica 68, $1303-1342$.

Sharpe, W. (2007), Investors and Markets: Portfolio Choices, Asset Prices, and Investment Advice, Princeton University Press.

Starr, M. (1973), 'Optimal production and allocation under uncertainty’, Quarterly Journal of Economics 87, 8195.

Vanayos, D. and Woolley, P. (2013), 'A institutional theory of momentumn and reversal', Review of Financial Studies 26, 1087-1145.

Wang, J. (1993), ‘A model of intertemporal asset prices under asymmetric information', Review of Economics and Statistics 60, 249-282.

Watanabe, M. (2008), 'Price volatility and investor behavior in an overlapping generations model with information asymmetry', Journal of Finance 63, 229-272.

Xiong, W. and Yan, H. (2010), 'Heterogeneous expectations and bond markets', Review of Financial Studies 23, 1433-1466. 
Yan, H. (2008), 'Natural selection in financial markets: Does it work?', Management Science 54, 1935-1950.

Yan, H. (2010), 'Is noise trading cancelled out by aggregation?', Management Science 57, 1047-1059.

Zapatero, F. (1998), 'Effects of financial innovations on market volatility when beliefs are heterogeneous', Journal of Economic Dynamics and Control 22, 597-626. 